\title{
The Stories of Logic and Information
}

Johan van Benthem (Amsterdam \& Stanford)

Maricarmen Martinez (Uniandes, Bogotá)

November 2007

Commentators

David Israel (SRI International)

John Perry (Stanford)

\section{$1 \quad$ Introduction and summary}

Information is a notion of wide use and great intuitive appeal, and hence, not surprisingly, different formal paradigms claim part of it, from Shannon channel theory to Kolmogorov complexity. Information is also a widely used term in logic, but a similar diversity repeats itself: there are several competing logical accounts of this notion, ranging from semantic to syntactic. In this chapter, we will discuss three major logical accounts of information.

Information as range The first notion is semantic, associated with sets of possible worlds, taken in a relaxed light sense, and we call it information as range. The greater one's range of options for what the real world is like, the less information one has. This setting reflects common sense ideas about information and uncertainty, but it also brings a more technical agenda of what information is good for - and we will develop some epistemic logic showing this. In particular, ranges of options change as agents make observations, or engage in communication. This process of 'update' high-lights a key feature of information in logic, and also in general: information is always to be understood in connection with some dynamic process using and transforming it. We will look at epistemic dynamic logics for this purpose, which form a natural calculus of changes in information ranges triggered by events of observation or communication.

Information as correlation The second major logical strand in information highlights another semantic feature, viz. that information tends to be about something that is relevant to us, and hence it crucially involves connections between different situations: my own, and others. This notion of information as correlation has been developed in situation theory, starting from a naturalist theory of meaning for agents living in information-rich physical environments, and moving to a more abstract view of components of a distributed system whose parts show 
dependencies. The correlation paradigm brings with it a further agenda of the structure of situations - well-founded or 'circular' -, ways of classifying them, constraints on their joint behaviour, and channels which allow for information flow. As for dynamic processes accessing this correlational structure, one can again think of observation or measurement or discourse, even though these have not been modeled in traditional situation theory per se.

Information as code Finally, there is a third major logical sense of information, oriented toward syntax, inference, and computation. This may be the primary sense for students learning logic and being told that valid conclusions 'add no information' to the premises. Thinking of information as encoded in sentences at some abstraction level, we come to the idea of information as code. In this concrete combinatorial setting, the major dynamic processing paradigm is 'inference' in some general sense, and the relevant logical sub-discipline is no longer model theory, but proof theory and theories of computation. Again dynamic processes are of the essence here, as both deduction and computation are stepwise activities of 'elucidation' which manipulate syntactic representations.

In all, then, we will see several static representations of information in logic, and several dynamic processes transforming them. In addition, further basic features come up in the relevant logical systems - in particular, the notions of 'aboutness': information is about something, and 'agency': information is for someone. As to the latter, logic has a rich account of attitudes and activities of agents, including their knowledge, beliefs, and activities of learning and revision over time. In an unpretentious diagram, a total view might be pictured as follows:

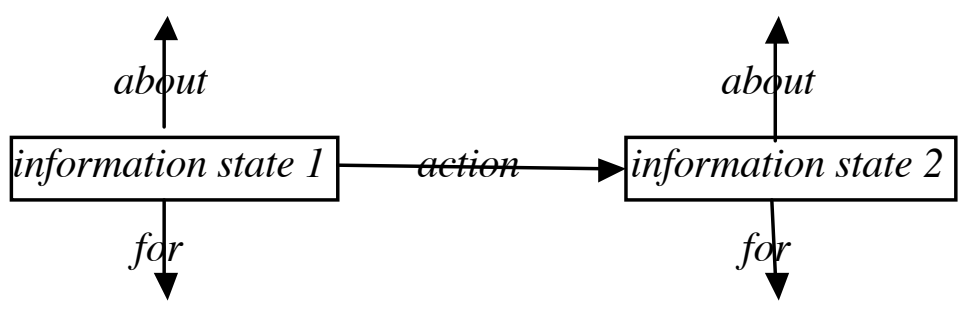

Co-existence versus unification This picture suggests that information as range and information as correlation are compatible semantic notions, and we show later how they can be merged, in the spirit of modern logics of dependence and interaction. Likewise, the co-existence of semantic and syntactic perspectives invites comparison. Semantic and syntactic perspectives have always co-existed in logic, and their interplay in capturing the same set of valid consequences is at the heart of the celebrated completeness theorems. We will show how this harmony also suggests links between more proof-theoretic information as code and 
semantic views of information and the processes transforming it, especially when we bring in the agents involved in these activities. Even so, this chapter provides no full-fledged grand unification of all bona fide logical notions of information. We even doubt whether one such unified notion is desirable, if it exists at all.

Beyond our horizon Much more could be said about logic and information, and we briefly list a few other topics at the end, including the increasing role of nonlinguistic visual (graphic) information carriers in logic, as well as the border-line between logic and quantitative approaches such as probability theory, which is spawning new hybrids today. But the above is our main agenda, and we refer to the literature for further historical and systematic background.

After this top-level sketch, let's plunge into concrete logical matters.

\section{Information in Logic}

Just a shaky metaphor? Information has a somewhat precarious status in logic. One uses it colloquially to explain to beginning students what logic is all about, and a favourite metaphor is then that deduction is useful for the purpose of 'extracting information' from the data at our disposal. Say, you know that $A \quad B$ and you learn that $\neg A$. Then logic tells you that you now know $B$, since the following basic inference schema is valid:

$$
A \quad B, \neg A / \neg B .
$$

By the way, this classic is also the schema behind Sudoku's and other logic puzzles sweeping the planet - so the reality of these phenomena of learning through logic is well-attested. But what one metaphor gives, another one takes. Further on in the course, we cheerfully tell students that the hall-mark of logical validity is its 'analyticity' in the sense of the classical philosophical tradition. And that says that the conclusions 'do not add information' to what was already there in the premises. ${ }^{1}$ Indeed, there are many different views of information in logic, but strikingly, all of them are implicit. The field has official definitions for its concepts of proof, computation, truth, or definability, but not of information! Indeed, many logicians will feel that this is significant. We do not need this notion

\footnotetext{
${ }^{1}$ This is close to validity in traditional logic, revived in the 'information-theoretic' account of Corcoran 1998. Saguillo 1996 discusses three views of consequence with their historical and philosophical background, casting the information-based one as a Third Way in addition to proof-theoretic and semantic views of validity.
} 
in the mechanics or even the foundations of the formal theory - or, as Laplace once said to Napoléon, who inquired into the absence of God in his Mécanique Céleste: "Sire, je n'avais pas besoin de cette hypothèse".

Information, inference, and computation Still, more can be said. There are several areas in modern logic where notions of information emerge naturally. We have already noticed the connection between information and inference, i.e., the proof-theoretic stance toward validity. Information states are then stages in a dynamic process of deduction, and the corresponding informational moves are proof steps, or more general computation steps. For a concrete illustration, think of successive stages in the solution of a $3 \times 3$ 'Sudokoid':

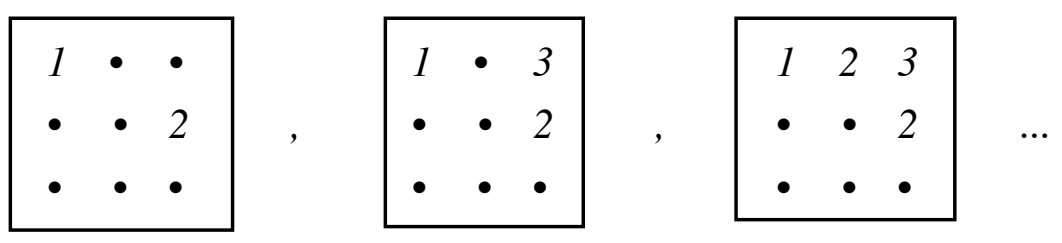

Intuitively, each successive diagram displays a bit more information about the eventual solution. Thus, on this view, information is brought to light in logical deduction or computation, and inferences are actions transforming the current state, i.e., the current representation structure appropriate to the topic of investigation. In the sequel, to coin a neutral phrase, we will sometimes refer to this dynamic use as elucidation.

Information range and update To find further logical notions of information beyond inference, however, consider just the simplest realistic scenario. Premises do not drop out of the sky! In a café, your friend has ordered Applejack, while you took a Brandy. A new waiter comes back from the kitchen with two glasses. What we see around us every day is that the new waiter asks who has the Applejack (say), puts it down, and then puts the other glass without asking. This scenario shows two informational processes intertwined. The final inference by the waiter produces information in the earlier sense, but so did your answer to his question, which was crucial to the whole information flow, be it in another sense. Your response did not involve deductive steps on your or his part, but rather changes in the waiter's information through observation, giving him new facts.

How should we model this second kind of information flow? Before learning the premises of the earlier inference, your information state contains 4 options. Receiving the first premise $A \quad B$ cuts this down to 3, and receiving the second premise $\neg A$ cuts down to just one option: $\neg A \& B$ is the actual situation. The rest 
seems indeed a matter of deductive inference, since a further update, say with the remaining fact $B$, would not change the final state any more:

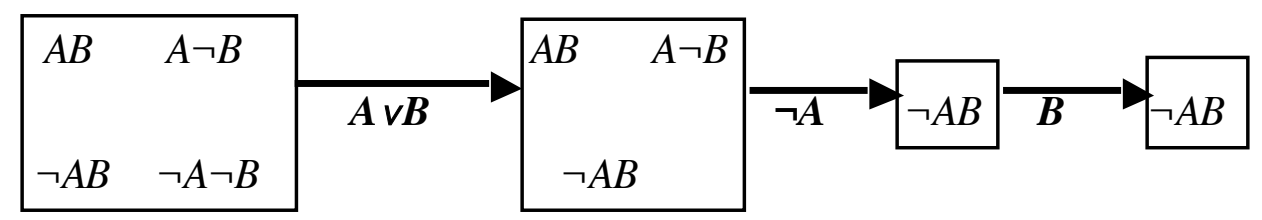

Again there is a process of information flow, this time through update. The resulting process is related to the semantic view of validity, which says that the conclusion must be true in all models for the premises. We do not know what the real situation is, but we have some information about it, encoded in a current range of models. And the relevant informational actions this time are updates: each subsequent assertion that we learn decreases that range, to a first approximation. This notion of information is found in epistemic logic and related semantic paradigms which we will discuss below in Sections 3 and 4.

Incidentally, note that in this second informational perspective, we are also extending the descriptive scope of logic. As the Restaurant example shows, inference is now just one of several information-producing processes entangled in even the simplest scenarios of information flow, and it seems tedious, and untrue to cognitive reality, to insist that 'logic' would just be about the inferential part, and not the rest. Indeed, there is even more to the scenario, as questions and answers involve processes of communication, another natural domain for logic.

Information and correlation But there are still further perspectives in our panorama! The research program which put information on the map perhaps most forcefully as a major theme for logical study per se was Situation Theory, the topic of Section 5 below. Here, flow of information is not seen primarily as driven by events of observation or communication between agents. It rather rests on the fact that we often learn about some situation that is not directly accessible to us via information at our disposal which is about that situation. Making sense of this aboutness calls attention to the 'constraints' in our environment, which correlate behaviour of spatio-temporally different situations. These constraints provide information channels between situations, and we can avail ourselves of these to draw conclusions about what we may not be able to observe directly.

Again, this correlational view may be tied in with notions of inference - once we are willing to step away from the usual varieties. E.g., one recurrent 'syllogism' in the ancient Indian logic tradition runs roughly as follows (Staal 1988). I am 
standing at the foot of the mountain, and cannot inspect directly what is going on there. But I can make observations in my current situation. Then, a useful inference might work as follows:

"I see smoke right here. Seeing smoke here indicates fire on the mountain. So, there is a fire on the mountain top." ${ }^{2}$

Compare this with the Aristotelean syllogism, which is about one fixed situation. The main new idea will be clear: logical inference can also cross between situations. Given suitable information channels between situations, observations about one may give reliable information concerning another. Indeed, the Indian example is almost the running example in Barwise \& Seligman 1997 on seeing a flash-light on the mountain in some by some observer safely in the valley. ${ }^{3}$

Incidentally, on the Indian view, reflected in parts of Western logic, inference is a sort of last resort, when other informational processes have failed. If I can see for myself what is happening in the room, that suffices. If I can ask some reliable person who knows, then that suffices as well. But if no direct or indirect observation is possible, we must resort to reasoning. Again, we see the entanglement of different informational processes mentioned earlier.

Information and interaction In this context of informational activities, it is worth noting that logic also has pragmatic perspectives beyond syntactic and semantic ones. Lorenzen 1955 famously explained a valid consequence as a dialogical claim in an argumentation game, whose proponent has a winning strategy against any opponent granting the premises. This is the world of Plato's "Dialogues" and public debate in the Greek polis, rather than the abstract proofs of Euclid's "Elements". On this pragmatic view we are after strategies which agents can use to win debating game or perform other interactive tasks. Going back in history, this stance also fits early views in Western and Chinese traditions on the role of inference in explanation to others, and the law of non-contradiction as the engine of debate (Liu \& Zhang 2007). This view also is in line with the earlier-mentioned scenario with questions and answers, which typically involved more than one agent. Thus, logic can also cast information as what flows in communication, and more generally, as the lubricant of intelligent interaction. We refer to the chapters by Devlin \& Rosenberg and by Walliser in this Handbook for more on this take,

\footnotetext{
${ }^{2}$ This version is simplified: Indian syllogisms had other interesting features.

${ }^{3}$ Other très Indian examples include observing a coiled object in a dark room, using logic, rather than touch, to find out if it is a piece of rope or a cobra.
} 
which fits well with current views of logic as a theory of interaction, with game theory as a natural ally. As with the earlier proof-theoretic and semantic views, we find information arising in a process, not as an absolute commodity by itself.

The goal of this chapter The major purpose of this chapter is to bring out information as a theme running all through logic, even when it is usually left implicit. The resulting take on logic today and some of its major features is shown in the following sections. This might seem mainly a matter of presentation and retelling existing stories. But there are genuine conceptual problems to be addressed as well. The many notions of information in logic pose a problem because they are so different - and yet they each clearly make some valid point. Thus the second purpose of this chapter is to provide a more unified perspective, pointing out some new connections, and raising some further questions. These issues have not been much addressed in the philosophy of logic, where the agenda still seems largely concerned with somewhat fossilized questions from the past.

To see that there is a potential for, if not unification, at least successful integration, we note that the above logical information theories have clear similarities. In particular, all involve an interplay between statics and dynamics. There are structures representing the information, but these only make sense as vehicles for various processes of information flow. Yet epistemic logic, situation theory, proof theory, and dialogue systems all approach this information dynamics from different stances. We think this diversity may be a blessing rather than a curse. We will elaborate on the stances, and discuss links between communication and correlation, or between observation and deduction. But we do not (yet) have one unified notion of information coming out of all this - and thus logic shows the same diversity one finds with the notion of information in general in this Handbook. Indeed, we will also high-light some connections to adjoining fields.

Finally, related issues to those discussed here occur elsewhere in this Handbook. The reader will find analogies in the chapters by Baltag, van Ditmarsch \& Moss on epistemic dynamics, Rot on belief revision, Kamp \& Stokhof on linguistic communication, and Abramsky on the information flow in computation.

\section{Information as range: state spaces, epistemic, and doxastic logic}

In this section, we develop the notion of information as range. In our view, current epistemic logic - broadly understood - is the 'information theory' that goes with this. We show how this brings along an agenda of further themes showing how 
this information functions, how it can be computed with, and what basic methodological issues then arise. The headings in our text identify what these are.

\subsection{Information, state spaces, and sentences}

Successive assertions inform us about a situation which the current discourse is about. Here is the natural semantic picture. It takes an 'inverse view' where extra information does not add things: it rather shrinks something, viz. the current range of options for what the real situation might be. We saw this with the earlier update pictures for the inference $A \quad B, \neg A / \neg B$, where the initial state of ignorance had 4 possible options, of which 3 remained after the input $A \quad B$, and only 1 after the further input of the premise $\neg A$. The inverse relationship is as follows, with $T$ for sets of formulas, and $M O D(T)$ for the class of models making all of $T$ true:

$$
T \square T^{\prime} \text { iff } M O D(T) \supseteq M O D\left(T^{\prime}\right)
$$

Using sets of models as information ranges is like the 'state spaces' used in BarHillel \& Carnap 1953 for describing the information associated with an assertion. But it would be silly to award a patent for this view to specific individuals. As we have said before, it seems close to the common sense through history.

Semantic sets of models are rather rough information states. A more finelygrained syntactic alternative would use languages describing properties of situations or worlds. Assembling assertions over time creates an ever-growing 'book' of sentences, the total current information - perhaps including inferential connections between sentences, and rankings as to relevance or plausibility. This syntactic view of the information at our disposal is natural, too, and it may be the most attractive from a computational point of view. Against this background, information as range provides only rough counterparts to information as sets of sentences, since $\operatorname{MOD}(T)=\operatorname{MOD}\left(T^{\prime}\right)$ for logically equivalent sets of assertions $T, T^{\prime}$, even when these are vastly different syntactically. To most logicians, this is a virtue, as they find 'details of syntax' irrelevant to content (as long as they are, one hopes, not reading love letters). Nevertheless, one can seek finer intermediate representations, and Carnap himself used syntactic 'state descriptions' for computations in his inductive logic. Lewis 1970 and Moore 1989 have lively discussions, going back to Russell, of how these issues play in logical semantics - but they have really exercised about every major philosophical logician, including Hintikka, Kripke, and Stalnaker. Indeed, much of the discussion of 'propositions' and 'meanings' in the philosophical literature (cf. the chapter by Kamp \& Stokhof in this Handbook) might be seen as the search for a level of information in between mere sets of models and every last detail of syntax. 


\subsection{Knowledge and epistemic logic}

We now discuss a setting which combines the semantic and syntactic perspectives. The best-known paradigm incorporating information as semantic range is epistemic logic, a subject proposed in Hintikka 1962, and developed by many authors since, across different disciplines such as philosophy, computer science, and economics. Its main ideas are easy to describe: models of the system describe information ranges for agents, while the matching language describes a notion of knowledge that can be paraphrased as "to the best of the agent's information". Here is how this works in more formal detail.

Language and models The syntax has proposition letters $p, q$, ..., Boolean operators $\neg, \quad$, and modal operators $K_{i} \square$. The latter say that agent $i$ knows that $\square$ while the dual $\left\langle i>\square=\neg K_{i} \neg \square\right.$ says that $i$ considers $\square$ possible. The following semantics provides a precise underpinning for this intuitive reading. Models $\boldsymbol{M}$ for the language are triples

$$
\left(W,\left\{\sim_{i} \mid i \square G\right\}, V\right),
$$

where $W$ is a set of worlds, the $\sim_{i}$ are binary accessibility relations between worlds, and $V$ is a propositional valuation recording in which world which atomic propositions are true. The worlds ('states', 'situations', ...) in the set $W$ represent the options for how the actual situation might be, while the relations $\sim_{i}$ encode the uncertainty, or alternatively, the current information of the agents:

$x \sim_{i} y$ says that, at world $x$,

$i$ considers $y$ an option for being the actual world.

These accessibility relations may be different for different agents, who evidently need not all have the same information. One often takes the $\sim_{i}$ to be equivalence relations, but this is not crucial to epistemic logic. ${ }^{4}$ Working with equivalence relations does validate some much-debated epistemic features of agents like 'positive' and 'negative introspection' concerning one's own knowledge. Now, the semantic truth condition for the knowledge operator makes the most evident stipulation in this setting, using a universal quantifier over the current information:

Agents know what is true throughout their current range of uncertainty:

$$
\boldsymbol{M}, s \mid=K_{i} \square \quad \text { iff } \quad \text { for all } t \text { with } s \sim_{i} t: M, t \mid=\square
$$

\footnotetext{
${ }^{4}$ Hintikka himself favoured mere reflexive transitive orderings.
} 
The dual $\left\langle i>\square=\neg K_{i} \neg \square\right.$ is then the existential quantifier 'in some currently accessible world', stating that agent $i$ holds it possible that $\square$ is the case.

We follow the usual 'knowledge' terminology for the operator $K_{i} \square$ of epistemic logic in much of what follows. Even so, as we have already said above, the more neutral and less ambitious term

'to the best of $i$ 's information' for $K_{i} \square$

states much better what the universal quantification over the current information range really achieves, and how epistemic logic can then serve as an information theory. We will briefly address this point of intended meaning again when discussing connections with formal epistemology.

Digression: alternative semantics for knowledge Possible worlds models have been under constant attack from critics. ${ }^{5}$ While some criticism seems merely a misguided response to the unfortunate 'worlds' metaphor for the situations represented in our models, there are respectable alternative traditions. Indeed, the earliest semantics for modal logic in the 1930s used topological models, reading $K_{i} \square$ as ' $\square$ is true throughout some open neighbourhood of the current point'. This stipulation represents another broad geometrical intuition about the structure of knowledge as range, generalizing the above graph-based models with accessibility arrows to include topological structures like the real numbers, or Euclidean space. Topological semantics for knowledge is undergoing a modest renaissance these days (van Benthem \& Sarenac 2005, van Benthem \& Bezhanishvili 2007), and generalized versions blossom in 'neighbourhood semantics' (cf. Chellas 1980, Arlo-Costa \& Pacuit 2005). ${ }^{6}$

Factual and higher-order information The epistemic language can formulate non-trivial scenarios. Consider a model for two agents $\boldsymbol{Q}, \boldsymbol{A}$ (say, 'questioner' and 'answerer') with one world where the atomic fact $P$ holds, and another where it fails. We assume that the real world (there always $i s$ one!) is the one indicated by the black dot - though this is of course an outsider's annotation, rather than the agents' own information. Labeled lines linking worlds indicate uncertainties. In particular, $\boldsymbol{Q}$ does not know which world is the actual one, while $\boldsymbol{A}$ is better

\footnotetext{
${ }^{5}$ This may explain why the paradigm is so vibrant and vigorous today.

${ }^{6}$ Moss, Parikh \& Steinsvold 2007 develop another type of epistemic logic based on topological ideas.
} 
informed: if the actual world has $P$ then she knows that is the case, and if it does not, then she knows that, too:

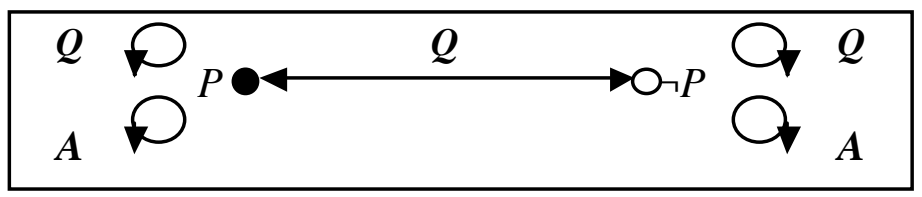

This diagram also encodes 'higher' information that agents have about their own and each others' information. For instance, in a precise sense to be defined below, $\boldsymbol{Q}$ knows that she does not know if $P$ while $\boldsymbol{A}$ does. Hence, it would be a good idea for $\boldsymbol{Q}$ to ask a question to $\boldsymbol{A}$, and find out. But before getting to that in Section 4 below, let us take stock of the model $\boldsymbol{M}$, representing the current state of the informational process. In formulas of the epistemic language, here are a few things which are true in the world to the left:

$$
\begin{aligned}
& P, K_{A} P, \neg K_{Q} P, K_{Q} \neg K_{Q} P, K_{Q}\left(\neg K_{Q} P \square \neg K_{Q} \neg P\right)(Q \text { knows that she does } \\
& \text { not know if } P), K_{Q}\left(K_{A} P \quad K_{A} \neg P\right)(Q \text { knows that } A \text { knows whether } P)
\end{aligned}
$$

Thus, this language of information can express complicated epistemic patterns.

In particular, the preceding iterations of knowledge about oneself and others reveal something essential about the notion of information as used by humans. 'Higher-order information' about (lack of) information of ourselves and others is just as important as ground-level factual information! By providing such iterations, our static language encodes crucial patterns of information in interaction. E.g., to see that mere knowledge about others' ignorance can be helpful, consider the following model, with the actual world at the black dot:

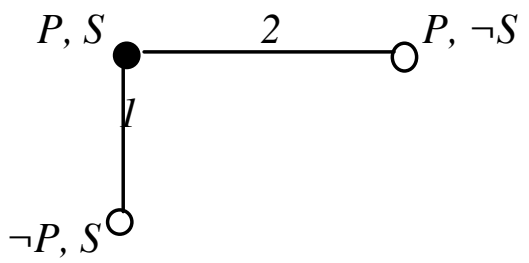

Given the uncertainty pattern, neither agent 1 nor 2 knows the real situation. But if 2 were to say she does not know, this is informative to 1 , as it rules out the bottom-most world. Hence, 1 learns the real situation, and can inform $2 .^{7}$

\footnotetext{
${ }^{7}$ In fact, in this setting, if both agents say simultaneously that they don't know, an epistemic model containing just the actual situation is reached at once.
} 
There is more to be said in logic about the information dynamics in these examples; and we shall do so in Section 3 below. For the moment, we just note that the above formal definitions alone do not really show the merits of a framework. Only a practical art of modeling can do that. And indeed, in the hands of skilled practitioners, epistemic logic is an information theory which handles much more complex scenarios than the baby examples here. Cf. Fagin et al. 1995, van der Hoek \& Meijer 1995, van Ditmarsch, van der Hoek \& Kooi 2007.

Model-theoretic digression: information and invariance In addition to its uses as a practical modeling device, epistemic logic raises general model-theoretic issues of expressive power. As in other formalisms, the crucial issue here is the harmony between expressive power and matching semantic invariances between models. In particular, the latter style of thinking raises the conceptual issue of

When are two given information models the same?

We cannot just take things at face value, and assume that geometrically different diagrams automatically model different information. To see this, consider the following variant $\boldsymbol{M}_{\boldsymbol{1}}$ of the epistemic model in the above scenario, with the actual world again indicated in bold-face:

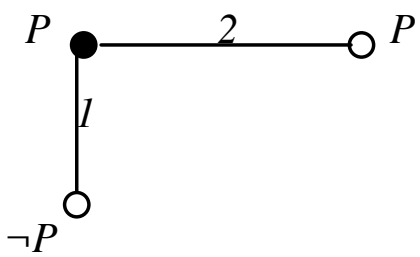

Now, let agent 2 announce the true fact that she does not know $\neg P$. The updated model will lose the lower-left world to become $\boldsymbol{M}_{2}$ :

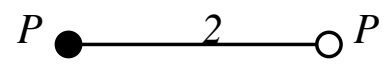

But clearly, $\boldsymbol{M}_{2}$ is essentially the same as the following one-world model $\boldsymbol{M}_{3}$ :

\section{$P$}

as can be seen by identifying both worlds in $\boldsymbol{M}_{3}$ with the single one in $\boldsymbol{M}_{3}$. Indeed, understanding what makes different diagrams carry 'the same' information is a good test of our understanding of the notion of information itself.

The standard semantic answer of epistemic logic is clear (van Benthem 1996, van Benthem \& Blackburn 2006). Two information models $(\boldsymbol{M}, s)$ and $(\boldsymbol{N}, t)$ satisfy 
the same epistemic formulas ${ }^{8}$ iff there exists a bisimulation between $\boldsymbol{M}$ and $\boldsymbol{N}$ connecting world $s$ to world $t$. We will not pursue the issue whether this is the only possible account of 'same information structure', but on our view, a true understanding of information should definitely come with a 'criterion of identity' allowing us to recognize the same information under different representations.

Having said this, we return to more standard and better-known issues.

Epistemic logic as information theory: two functions Any logical system has at least two major functions. First, its language can state properties of situations, and hence it has descriptive and communicative uses, just like a natural language. And once we have stated such properties, we can check computationally whether they hold in specific models, or we can compute concrete updates of the current model.

But there is also a second use for a logic, as a description of valid inference, supplying in our current case a 'calculus of information'. This second function comes ideally in the form of complete syntactic calculi describing all valid principles of inference with knowledge, such as multi-agent ' $K$ ' or ' $S 5^{\prime}$ ' or other well-known axiomatic systems. E.g., here is the complete set of principles for S5, on top of any complete classical propositional logic:

$$
\begin{array}{ll}
K_{j}(\square \square \square) \square \quad\left(K_{j} \square \square K_{j} \square\right) & \text { Knowledge Distribution } \\
K_{j} \square \square \square & \text { Veridicality } \\
K_{j} \square \square K_{j} K_{j} \square & \text { Positive Introspection } \\
\neg K_{j} \square \square K_{j} \neg K_{j} \square & \text { Negative Introspection }
\end{array}
$$

The complete logic with all the above principles is decidable, be it computationally more complex than its propositional base. It is 'Pspace-complete', rather than 'NP-complete', giving it the same complexity as many games (Papadimitriou 1994) - again an indication of its interactive character. These laws of inference can be applied in every concrete scenario, just like the principles of probability theory. This axiomatic system describes both the agents' own reasoning, and our external reasoning as theorists about them - though the distinction has been played up in the recent literature. We will ignore that issue here, but we do briefly discuss a few of these axioms below, since they are not just rock-bottom truths: they reflect a particular view of powers of epistemic agents.

\footnotetext{
${ }^{8}$ in an infinitary version of the epistemic language whose technical details are irrelevant here .
} 
Altogether then, epistemic logic can be used to describe both information update and inference, making it serve the broader purposes of logic as a theory of different kinds of information advocated in Section 2.

\subsection{Other attitudes: doxastic and conditional logic}

In the above, we have noted a separation between information per se coming from some source, and agents' attitudes and responses to it. Indeed, there are many attitudes that agents can have toward propositions beyond knowledge. Our natural language has a fine-grained repertoire, from knowing propositions to believing, or even just 'entertaining' them. Moreover, we can also doubt propositions, maybe on the basis of new information - and more generally, change our allegiance from one epistemic attitude to another. Some of these ubiquitous attitudes toward information have received formal treatment in logic.

Basic doxastic logic In particular, 'doxastic logics' analyze assertions $B_{i} \square$ standing for 'agent $i$ believes that $\square$. The semantics for the belief operator used in this chapter seems folklore. It adds a new idea to the mere ranges for agents in our epistemic models so far. We now assume further semantic structure, in the form of a plausibility ordering of worlds as seen from some vantage point:

$$
\leq_{i, s} x y \quad \text { in world } s \text {, agent } i \text { considers } y \text { at least as plausible as } x \text {. }
$$

Thus, while the earlier ranges of alternatives corresponded to the strict information that agents have, these same ranges ordered by plausibility give much finer gradations. In particular, we can now define belief semantically as less demanding than knowledge, in the sense of 'truth in the most plausible options':

$$
\boldsymbol{M}, s \mid=B_{i} \square \text { iff } \boldsymbol{M}, t \mid=\square \text { for all } t \text { maximal in the ordering } x \leq_{i, s} y .
$$

Here is an elementary example. Consider a model $\boldsymbol{M}$ with two worlds that are mutually epistemically accessible, but the one with $\neg P$ is considered more plausible than the other with $P$ (which happens to be the actual world):

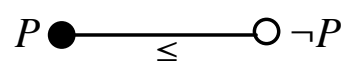

In this model, at the actual world where $P$ holds, the agent does not know if $P$, but she does (mistakenly!) believe that $\neg P$. It is crucial that our beliefs can be false. ${ }^{9}$ As with epistemic logic, there are complete doxastic logics, and a further theory around them (Fagin et al. 1995). In general the resulting logics also analyze the

\footnotetext{
${ }^{9}$ There are some technical complications in making this semantics work in infinite models, which we ignore here.
} 
interplay between knowledge and belief, with knowledge implying belief, and more controversially, taking a stance on such issues as whether one knows one's beliefs, and so on. While inter-relations between attitudes toward information are an important topic in logic, we omit it here as tangential to our main concerns.

Digression: information or 'attitude'? We can think of these epistemic-doxastic information models with two relations $\sim_{i}, \leq_{j}$ in two ways. The orderings $\leq_{i, s}$ may just encode an agent's private response to the strict information received, making the belief subjective. But we can also view them more objectively as encoding intrinsic gradations of plausibility in the incoming information - with beliefs the mere registering of this by sensitive logical observers. Both interpretations occur in the literature, like with probability - and both have their uses.

Conditional doxastic logic In doxastic logic as used in dynamic informational processes, one soon finds that mere beliefs are not sufficient for explaining agents' behaviour over time. We want to know what they would do in certain scenarios where they receive new information. This requires conditional belief:

$\boldsymbol{M}, s \mid=B_{i}^{\square} \square \quad$ iff $\quad \boldsymbol{M}, t \mid=\square$ for all worlds $t$ which

are maximal for $x \leq_{i, s} y$ in the set $\{u|M, u|=\square\}$.

Conditional beliefs $B_{i}^{\square} \square$ are like logical conditionals in general, expressing what might happen under different circumstances from where we are now. Thus they pre-encode beliefs in $\square$ we would have if we were to learn new things $\square$. The analogy is so close that conditional belief on reflexive transitive plausibility models satisfies exactly the principles of the minimal conditional logic (Burgess 1981, Veltman 1985). We will sharpen things up when discussing the mechanics of belief revision in Section 4.

\subsection{Agents: powers, attitudes, and collective information}

Epistemic logic highlights three aspects of our schematic diagram of information flow in Section 1. It is about information states (the above semantic models) and information dynamics - but crucially, always as performed by informationhandling agents. We have already seen several aspects of this agent-orientation. Information is relative to agents, and they can have a spectrum of different attitudes toward it: knowledge, belief, and others. In addition, agents can also have very different abilities - with some choice-points high-lighted in the axioms of epistemic logic. For instance, the Distribution Axiom $K_{j}(\square \square \square) \square \quad\left(K_{j} \square \square K_{j} \square\right)$ says that knowledge is closed under known implications, giving agents unlimited powers of stepwise inference from what they already know. Likewise, the 
Introspection Axioms give them unlimited powers of self-reflection on what they know or do not know. These abilities of 'omniscience' and 'introspection' have been questioned in much of the philosophical and computational literature though no alternative consensus model of 'bounded rationality' has emerged so far. Still, epistemic systems today provide for agent variation. Now this raises an issue, and perhaps also a fact of life.

Agent relativity and agent diversity Agent-relativity seems to dilute the absoluteness of a bona fide information theory. But it may be a genuine insight. Our account of 'knowledge' as 'to the best of an agent's information' really shows that there is an unavoidable interplay between two notions which needs to be made explicit: (a) the information in a situation per se, and (b) the powers of the agents having access to it. Put as a general principle, to see what information is available, one must take the informational nature of the agents into account.

Existing epistemic logics differ on what they ascribe to agents (van Benthem \& Liu 2004, Liu 2006) in terms of deductive powers, introspective abilities, observational powers, and memory capacity. Such assignments are reflected in correspondences between such powers of agents and axioms of the logic. Examples are the much-discussed link between the ' $K K$ principle' $K \square \square K K \square$ and transitivity of accessibility, or between commutation laws for communication (cf. Section 5) and memory assumptions of perfect recall. These correspondences provide a refined logical view of what information can flow in a given setting, given the nature of a source and that of the recipient.

Group knowledge and social structure Our final observation about agent orientation in epistemic logic is its social character. This showed in the interactive nature of knowledge about others, but it goes much further than this. Agents also form groups, as in the above question-answer scenario, where communication involves a group consisting of the participants, which can have knowledge of its own. In particular, epistemic logic also has notions of group knowledge which are sui generis, with pride of place going to

\section{$C_{G} \square\left(' \square\right.$ is common knowledge in group $\left.G^{\prime}\right)$,}

which is read in the above information models as follows:

$\boldsymbol{M}, s \mathrm{I}=C_{G} \square$ iff for all worlds $t$ reachable from $s$ by some finite sequence of $\sim_{i}$ steps $(i \square G): M, t \mid=\square$.

In the logic, the additional axioms for $C_{G}$ bring out the 'reflexive' fixed-point character which common knowledge has intuitively: 


$$
\begin{array}{ll}
C_{G} \square \square \quad \square \& E_{G} C_{G} \square & \text { Equilibrium Axiom } \\
\left(\square \& C_{G}\left(\square E_{G} \square\right)\right) \square C_{G} \square & \text { Induction Axiom }^{10}
\end{array}
$$

Another natural form of group knowledge is 'distributed knowledge' referring to what a group might come to know if it pooled the information available to individual members (cf. Fagin et al., 1995, van Benthem 2006B). The same social group notions occur in the literature for belief.

\subsection{Connections with other fields}

Philosophy Epistemic-doxastic logic is a somewhat austere account of qualitative information. But ever since its birth, it has triggered discussions in philosophical epistemology. It was soon found that the universal quantifier in information as range provides a rather poor analysis of knowledge in the philosopher's demanding sense, where the quest for a satisfactory definition of knowing that $P$ involves finding the right sort of 'robustness' in addition to the obvious features of truth of $P$ and belief in $P$. Plato famously proposed 'justified true belief', but sophisticated new definitions have kept appearing until today, such as Dretske's 'true belief grounded in correct information', or Nozick's 'true belief with counterfactual backing: if $P$ had been false, we would have believed that $\neg P^{\prime}$. Even though epistemic logic has never offered a definition of knowledge of comparable sophistication, the very mismatch with the richer philosophical tradition has been much more exciting than many happy marriages, leading to clarification and fruitful debate. For instance, the distribution axiom $K(\square \square \square) \square(K \square \square K \square$ ) has sparked debates about Logical Omniscience, and the axiom $K \square \square \quad K K \square$ about Positive Introspection. We refer the reader to Hendricks 2006, Williamson 2000, van Benthem 2006A, Baltag, van Benthem \& Smets 2007 for a wide array of interface topics, such as definitions of knowledge, skeptical arguments (see also Dretske's chapter in this Handbook), different sources of information (language, senses, etc.), omniscience, bounded rationality, and reflection. ${ }^{11}$

Computer science, and economics But maybe the philosophers set their standards of knowledge too high. Who searches for the Perfect Knight might never get married. For many practical purposes, the picture of knowledge as range

\footnotetext{
${ }^{10}$ Here $E_{G} \square$ says that everyone in the group $G$ knows that $\square$.

${ }^{11}$ Many of these issues are high-lighted by persistent puzzles, such as infelicitous Moore sentences, the Fitch Paradox, or the Ramsey Test.
} 
seems quite sufficient. Think of scenarios like this. The cards have been dealt. I know there are 52 of them, and I know their colours. The possible worlds are just the possible deals. Of course, I could be wrong about this ${ }^{12}$, but this worry seems morbid, and not useful in understanding normal information flow. What is true is that less well-founded attitudes will come in as well. I may only have ephemeral beliefs about who holds which card, or about how the other agents will play. And indeed, we are sensitive to such distinctions. 'Knowledge' may then be a contextdependent term for 'the strictest attitude in the current setting'.

Notions of knowledge based on epistemic logic have penetrated other areas, notably computer science, witness the interdisciplinary TARK conferences since the early 1980s (Fagin et al. 1995), with their current companion event LOFT. Originally, knowledge was ascribed here metaphorically to processors in distributed systems, with accessibility arising as these can only distinguish global system states through their own local state. But in modern intelligent agent-based computing systems, the difference with humans seems slight. Also, and even somewhat earlier, epistemic logic entered economic game theory in the 1970s through the work of Aumann (cf. Osborne \& Rubinstein 1994, de Bruin 2004), as a way of stating what players know about each other, in an account of the reasoning about rationality underpinning for notions like Backward Induction and Nash Equilibrium. Cf. van der Hoek \& Pauly 2006 for further details, as well as the chapter by Walliser in this Handbook.

In applications such as these, knowledge often occurs intermingled with moves or actions. Players reason using their knowledge of what certain moves will bring about, and also, after observing a move by other players, they readjust their current information. This natural combination will be the topic of the next section.

\section{$4 \quad$ Information flow and dynamic logic}

Communication and information flow We start by summarizing a point which pervaded much of the preceding Section. As in other information theories, such as Shannon's quantitative account of channel transmission (cf. the chapters by Harremoes \& Topsoe and by Grunwald \& Vitanyi in this Handbook), information really comes into its own only in a dynamic setting of communication and information flow. As a simplest case of this phenomenon in epistemic logic, consider the following conversational scenario:

\footnotetext{
${ }^{12}$ Perhaps someone replaced the King of Hearts by Bill Clinton's visiting card.
} 
(a) $\quad \boldsymbol{Q}$ asks $\boldsymbol{A}$ the question "P?",

(b) $\boldsymbol{A}$ gives the true answer "Yes".

Then the situation depicted in the model $\boldsymbol{M}$ changes, since information will now come to flow. If the episode is one of simple cooperative Gricean communication, the question (a) itself conveys the information that $\boldsymbol{Q}$ does not know the answer, but also, that she thinks $\boldsymbol{A}$ might know. In general, this iteration can be highly informative and useful to the answerer. Next, the answering event (b) conveys that $\boldsymbol{A}$ knows that $P$, and its public announcement in the group $\{\boldsymbol{Q}, \boldsymbol{A}\}$ makes sure that $Q$ now also knows that $P$, that both agents know this about each other, and so on to very depth of iteration. In terms of the philosophical classic Lewis 1969, after the episode, the agents have achieved common knowledge of $P$.

Structure and process Summing up, our earlier point returns. It is hard to think of information in isolation from the processes which create, modify, and convey it. This combination of structure and process is quite natural in many disciplines. In computer science, one designs data structures in tandem with the processes that manipulate them, and the tasks which the latter should perform. And the same point is familiar from philosophy, as in David Lewis' famous dictum that 'Meaning Is what Meaning Does'. We can only give good representations of meanings for linguistic expressions when we state how they are going to be used: in communication, disambiguation, inference, and so on. In a slogan:

Structure should always come in tandem with a process!

So, which dynamic processes drive the notion of information? Many candidates vie for this honour in the chapters of this Handbook, including computation, inference, update, revision, correction, question answering, communication, games, and learning. Some items in this list are activities of single agents, while others are intrinsically interactive 'social' phenomena - with interesting connections between the two. We will not investigate all these links in detail, or even the issue whether one notion of information serves them all. ${ }^{13}$ Instead, we will highlight one instance of this Dynamic Turn, in terms of informational processes for epistemicdoxastic logic. The chapters by Baltag, van Ditmarsch \& Moss and by Rott in this Handbook provide much further elaboration.

Information update and model change One of the simplest events providing new information is a public announcement!P of some fact $P$. One can think of

\footnotetext{
${ }^{13} \mathrm{Cf}$. the general Editorial to this Handbook for further thoughts on this issue.
} 
this as a statement coming from some authoritative source - or more generally, as a totally reliable observation based on one's senses. If I see that the Ace of Spades is played on the table, I now come to know that no one of us holds it any more. ${ }^{14}$ These events of new hard information in the earlier sense change what I know.

Formally, this happens by their triggering changes in the current epistemic model. In particular, public announcements !P work as described in Section 1 above. They eliminate all worlds incompatible with $P$, thereby zooming in on the actual situation. Thus the current model $(\boldsymbol{M}, s)$ with actual world $s$ changes into its submodel $(\boldsymbol{M} \mid P, s)$, whose domain is the set $\{t \square \boldsymbol{M}|\boldsymbol{M}, t|=P\}$. In a picture, one goes

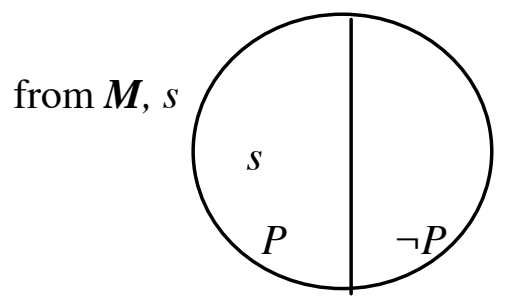

to $\boldsymbol{M} \mid P, \mathrm{~s}$

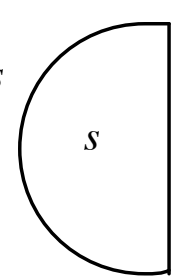

Dynamic logic of public announcement Crucially, truth values of epistemic formulas may change in such an update step: typically, agents who did not know that $P$ now do after the announcement. One can keep track of this epistemic dynamics in so-called public announcement logic PAL, extending the epistemic language with action expressions in a two-level syntax:

$$
\begin{array}{lll}
\text { Formulas } & P: & p|\neg \square| \square \square\left|K_{i} \square\right| C_{G} \square \text { । }[A] \square \\
\text { Action expressions } & A: & ! P
\end{array}
$$

The semantic clause for the dynamic action modality is as follows:

$$
\boldsymbol{M}, s \mid=[! P] \square \quad \text { iff } \quad \text { if } \boldsymbol{M}, s \mid=P \text {, then } \boldsymbol{M}|P, s|=\square
$$

As all this is less known than standard epistemic logic, we state the principles of the complete calculus of information flow under public announcement. It has the usual laws of epistemic logic over one's chosen base models (as discussed earlier) plus, crucially, the following reduction axioms:
(a) $[! P] q$
$\square \quad P \square \quad q$
for atomic facts $q$
(b) $\quad[! P] \neg \square$
$\square \quad P \square \neg[! P] \square$
(c) $\quad[! P](\square \square \square) \quad \square$
$[! P] \square \square[! P] \square$
(d) $\quad[! P] K_{i} \square$
$P \square K_{i}(P \square[! P] \square)$

\footnotetext{
${ }^{14}$ Of course, we can be mistaken about what we hear or see, but overly morbid worries can block the progress of logic.
} 
Taken together, these principles allow for compositional analysis of what agents know after new hard information has come in. In particular, the equivalence (d) is a dynamic 'recursion equation' relating knowledge before and after the event !P. This is the earlier idea of pre-encoding. There is already sufficient information about the effects of events $! P$ in the current state through the relativized knowledge of the form $K_{i}(P \square$. In order to find similar recursion equations for other informational events, and other attitudes of agents such as belief, similar expressive demands will have to be met by the static language. ${ }^{15}$

Agent powers again Logical axioms high-light basic issues in concentrated form. The dynamic knowledge axiom $[! P] K_{i} \square \square\left(P \square K_{i}(P \square \quad[! P] \square)\right)$ also involves an interchange between the dynamic operator $[! P]$ and the epistemic $K_{i}$. This commutativity is not obvious, witness its possible failures in general logics of knowledge and action (Moore 1985). I know now that after drinking I am boring. But the tragedy of my life is that, after drinking, I do not know that I am boring... The reason is that drinking, unlike mere observation, impairs my epistemic abilities. Knowledge action interchanges presuppose abilities of agents such as Perfect Recall (Halpern \& Vardi 1989, van Benthem 2001). Again we see that studying information flow immediately raises issues of agent ability.

General observation and event update Public announcement or observation is just one process which produces information. More complex changes occur in the current epistemic model for a group of agents when parlour games are played, or emails are sent with 'hidden buttons' like bcc. The relevant changes high-light another power of agents which admits of variation. This time, the issue is not inferential zeal, introspection, or memory, but agents' potentially limited and diverse powers of observation. Say, I see you draw a card from the stack, but unlike you, without seeing exactly which one. So, how to model this varying observational access? The answer is a non-trivial generalization of public update:

In general dynamic-epistemic logics (Baltag, Moss \& Solecki 1998, Gerbrandy 1999, van Benthem, van Eijck \& Kooi 2006, van Ditmarsch, van der Hoek \& Kooi 2007), so-called event models $\boldsymbol{A}$ describe complex scenarios where not all agents have the same observational access to what is happening. This leads to a

\footnotetext{
${ }^{15}$ As an extended modal logic, $P A L$ still has a bisimulation-based model theory, and it raises many interesting new issues of expressive power and computational complexity (cf. van Benthem 2006B).
} 
mechanism of product update, turning the current epistemic model $\boldsymbol{M}$ into a model $\boldsymbol{M} \boldsymbol{x} \boldsymbol{A}$ which can even be larger than $\boldsymbol{M}$ itself, recording information of different agents about base facts and what others know. Product update redefines the universe of relevant possible worlds, and also the epistemic accessibility relations between them. Conversations, games, internet transactions, and other real activities are like this. For this natural continuation of our present analysis, we refer to the chapter by Baltag, van Ditmarsch \& Moss in this Handbook. ${ }^{16}$

Hard and soft information But there is more to information flow than hard information, whether observed totally or partially, and the resulting knowledge. As we saw in Section 4, further fine-structure may be present on epistemic models through plausibility orderings. These can be viewed as representing agents' attitudes, or as the result of receiving what might be called soft information. This plausibility ordering is reflected in the informational attitude of belief, absolute or conditional, telling us - roughly - what agents would believe when confronted with new hard information. And in the end, it is the total interplay of all these attitudes which would describe our stances toward information, and the way they are affected by new incoming information. Thus, we get a mixture of information per se, and the ways in which agents take it - and this logical entanglement is such it is hard to say where one notion ends and the other begins. ${ }^{17}$

Changing beliefs Now we are ready for the next obvious step. The dynamic process perspective on information change explained here also applies to the doxastic attitude of our beliefs, and how to revise these on the basis of incoming information (Gärdenfors 1987). This involves changes, not in the range of available worlds or in their epistemic accessibility patterns, but rather in the earlier plausibility orderings $x \leq_{i, s} y$ among worlds.

How this works precisely, depends on the incoming signal. When we receive hard information !P, update will proceed by world elimination as before. We then get

\footnotetext{
${ }^{16}$ Systems with similar aims, but with richer languages allowing for process description in the long run over time, are epistemic-temporal logics, developed by Gupta \& Thomason, Belnap, Perlof \& Ming Xu, Fagin, Halpern, Moses \& Vardi, Parikh \& Ramanujam, and other authors. Van Benthem \& Pacuit 2006 and van Benthem, Gerbrandy \& Pacuit 2007 give surveys and comparisons.

${ }^{17}$ Other views distinguish between a base level of pure information processing and a higher level of beliefs on the basis of this information, where belief changes occur in a sort of 'reflective dynamics'. Cf. the chapter by Rott in this Handbook.
} 
new beliefs related to our earlier conditional beliefs, and the counterpart to the above reduction axiom (d) is the following recursion equation saying which new beliefs - and indeed, conditional beliefs - are acquired (van Benthem 2007A):

$\begin{array}{llll}\text { (e) } & {[! P] B_{i} \square} & \square & P \square B_{i}{ }^{P}[! P] \square \\ \text { (f) } & {[! P] B_{i}{ }^{\square} \square} & \square & P \square B_{i}{ }^{P \square[! P] \square}[! P] \square\end{array}$

But often, we just get soft information signals of the sort mentioned before. These increase our 'preference' for $P$-worlds, but without telling us to abandon the others. A quite typical 'belief revision policy' in this spirit is lexicographic upgrade $\square P$ (Rott 2006) which replaces the current ordering relation $\leq$ between worlds by the following:

all $P$-worlds become better than all $\neg P$-worlds,

and within those two zones, the old ordering remains.

Belief changes under such policies can again be axiomatized completely (cf. again van Benthem 2007A). Just for illustration, here is the recursion equation for conditional beliefs following the $\square P$ revision policy. It looks somewhat forbidding - but perhaps necessarily so: after all, we are now describing a more complex informational process than mere epistemic update:

$$
\begin{aligned}
{[\square P] B^{\square} \square \square \quad\left(<>(P \square[\square P] \square) \square B^{P \square[\square]}[\square P] \square\right) } & \left(\neg\left(<>(P \square[\square P] \square) \square B^{1\left[\square^{P}\right] \square}[\square P] \square\right)^{18}\right.
\end{aligned}
$$

Still richer dynamic doxastic logics use the above event models as triggers for belief revision, with 'signal events' ordered by plausibility (Baltag \& Smets 2006). This relocates revision policies in the structure of the incoming signal, while sticking to one product update rule for the new plausibility ordering. We refer to the chapters by Baltag, van Ditmarsch \& Moss and by Rott in this Handbook for further policies, and deeper discussion of the issues raised by all this. ${ }^{19}$

Information-processing agents: the general setting The over-all view of information dynamics which emerges from our discussion of information update and belief revision in Sections 3, 4 is quite elaborate. Agents have powers of inference, observation, introspection, and self-correction, maybe not all to the same

\footnotetext{
${ }^{18}$ Here the existential epistemic modality <> in the consequent says the assertion is true 'in some world of the current range'.

${ }^{19}$ There are also strong analogies between processes of plausibility re-ordering and recent dynamic logics of preference change (van Benthem \& Liu 2007).
} 
degree - and moreover, they exercise these powers interactively, in groups of agents, leading to behavioral equilibria that naturally invite study per se. This picture is in line with broader current trends. These include 'logical dynamics' (van Benthem 1996), 'information dynamics' in computer science (cf. Abramsky's chapter in this Handbook) and 'interactive epistemology' in philosophy and game theory (cf. the chapter by Walliser). There are also connections with mathematical learning theory (Kelly 1996, and also his chapter in this Handbook).

Here we note once more that our logical systems have the following features as 'information theories'. They are dynamic, treating informational actions and events on a par with static information structures. They are also social, in that basic scenarios have more than one agent together. ${ }^{20}$ Thus interaction between agents, and even irreducible group action and group knowledge, become crucial to a logical understanding of information flow.

\section{Information as Correlation: the world of Situation Theory}

We now turn to the only program in modern logic that treats information as its central notion, shaking up the usual agenda of research. It originated in the 1980's (Barwise \& Perry 1983) as a foundational framework for 'situation semantics', but in its underlying situation theory, the program has come to study the notion of information from a much more general point of view (cf. also Devlin 1991, Allo 2007, and the chapter by Devlin \& Rosenberg in this Handbook).

According to situation semantics, meaning arises from the interaction of organisms and their information-rich environment. As a formal tool originally devised for modeling this view of meaning and associated phenomena, situation theory goes beyond possible-worlds models by providing richer structure. Among other things, this yields a natural treatment of 'the problem of grain': different false claims about the world are represented by different mathematical objects. In addition, the theory provides an account of important context effects that are part of everyday inference and natural language.

The central role of the notion of information in situation theory, and the way it was formalized, reflects several influences. For example, like the Gibsonian view of reality and its role in visual perception (Gibson 1979), in situation theory everything is part of as a structured reality which is full of 'uniformities'. Organisms are 'attuned' to those regularities, and that allows them to survive.

\footnotetext{
${ }^{20}$ Even observation is really a matter between 'me' and 'my source'.
} 
Information is a pervasive aspect of reality, prior to cognitive action. Other important influences came from philosophy. For example, Putnam's Twin Earth thought experiment (Putnam 1981) has convinced many that meaning cannot be just in the mind, and hence external reality must play a crucial role. But it is Dretske's theory of information flow (Dretske 1981) that conspicuously provided key ideas about the basic informational notion to study. Dretske builds on Shannon's theory of information transmission, but his account is qualitative and his focus is squarely on semantic content, not on the amount of information transmitted. It uses the following variant of the usual notion of confirmation:

Information Content To an agent with prior knowledge $k$, signal $r$ carries the information that $s$ is $F$ iff $\operatorname{Pr}(s$ is $F \mid r$ and $k$ ) $=1$, but $\operatorname{Pr}($ s is $F \mid k)<1$.

Dretske then defines knowledge as belief caused by information flow. One reason why the definition includes the strict equality $\operatorname{Pr}(s$ is $F \mid r$ and $k)=1^{21}$, is the following intuitively appealing consequence:

Xerox Principle If $A$ carries the information that $B$ and $B$ carries the information that $C$, then $A$ carries the information that $C$.

Situation theory adopts the same guiding idea - though dropping the probabilities - , and it encompasses not only natural but also conventional signals and others.

Furthermore, in situation theory we distinguish between having and carrying information. This distinction is reflected in the study of two kinds of reports in natural language. Epistemic attitude reports report the information an agent has:

(1) Elwood knows that Santa Cruz is east of Berkeley

(2) Elwood sees that Gretchen is limping.

In contrast to this, information reports tell what is shown or indicated by an event or the state of an object (the signal[:

(3) The $x$-ray's being of pattern $\square$ shows that Gretchen has a broken leg.

(4) The array of pixels on the screen being of its specific pattern carried the information that the Forty-niners had won the championship.

In (3) the $x$-ray having its specific pattern is the indicating fact; the $x$-ray itself is the carrier of the information.

\footnotetext{
${ }^{21}$ This stipulation has been the target of some criticisms, which we disregard here.
} 
Treatments of information as correlation as mentioned in our Introduction focus on information reports, in which one state of affairs carries information about another; the way one thing indicates how another thing is; the way the $x$-ray turned out carries information about Gretchen's leg. More generally, one situation carries information about another situation connected to it in some way, by some channel of information, in virtue of a regularity or constraint: perhaps a natural law, a convention, or something else. By contrast, epistemic attitude reports about having information are closer to the epistemic logic range view discussed before.

We now come to the basic apparatus, which we describe in enough detail to contrast it with the preceding sections. Still, the thrust will differ somewhat. Epistemic logic in its static and dynamic versions has a well-developed model theory and proof calculus, but it provides little information at a practical level about what might be called the 'art of modeling'. Situation theory does a kind of converse. It offers a rich apparatus for modeling challenging phenomena - but so far, it has (with a few exceptions to be noted below) not yet generated canonical calculi of reasoning with the same sweep and wide application as epistemic logic.

\subsection{Basic concepts of situation theory}

Situation theory starts from a concrete reality, with concrete parts but no concrete alternatives. This reality can be thought about, perceived, studied and analyzed in a variety of different ways, from different perspectives, for different purposes. But ultimately everything that exists, everything that happens, everything that is true, has its status because of the nature of this reality. The parts of reality are what we call situations. Situation theory is committed to there being situations, but need not be committed to there being a largest total situation of which the rest are parts.

States of affairs When we think or talk about reality, we need some way of analyzing it. This we call a system of classification and individuation. It consists of situations, relations, locations and individuals. The commonplace that different schemes can be used to study the same reality is one to which situation theory subscribes. But this does not make situations structure-less, with properties projected onto them by language or thought. Rather, situations are rich in structure, and support a variety of schemes, suited (or unsuited) to various needs.

Each relation $R$ comes with a set of argument roles. For example, the relation of eating comes with the roles of eater, eaten, and the location of eating. Objects of appropriate sorts play these roles. A relation, together with objects assigned to its roles, gives rise to an issue, namely, whether or not the objects stand in the 
relation. There are two possibilities, and each of these we call a state of affairs. For example, if eating is the relation, Bush is the eater, a certain quantity of succotash is the eaten, and the White House at a certain time is the location (call it $l o c)$, then there are the following two states of affairs:

$<$ Eats; loc, Bush, the succotash; 1 >>,

$<<$ Eats; loc, Bush, the succotash; 0>>

The issue is whether Bush eats the succotash at the location; the possible answers are 'yes $\square(1)$ and 'no $\square(0)$. The first state of affairs resolves the issue positively, the second, negatively. We say the first has a positive and the second a negative polarity. Each of these two is the dual of the other.

Although we don't assume that the argument roles of a relation have a natural order, we often use the order suggested by English to identify argument roles, without explicitly mentioning the roles themselves, as we did above.

Facts and the partiality of situations Situations determine whether a given state of affairs or its dual is a fact. As for notation,

$s \mid=\square$ means that $s$ makes $\square$ factual, or s supports $\square$

and

$I=\square \quad$ means that $\square$ is factual (i.e., some real situation supports it)

Given a state of affairs $\square$, these are uncontroversial theses about the $\mathrm{l}=$ relation:

1. Some situation will make $\square$ or its dual factual,

2. No situation will make both $\square$ and its dual factual,

3. Some situations will leave the relevant issue unresolved, making neither $\square$ nor its dual factual.

In contrast, the following is a controversial thesis about this important relation:

4. Some situation resolves all issues (i.e., there is a largest total situation).

The third thesis says that situations are partial. Hence, there are two ways a situation $s$ can fail to make a given state of affairs $\square$ factual. Namely, $s$ may make the dual of $\square$ factual, or $s$ may fail to resolve the $\square$-issue one way or the other.

Parameters, anchors, and infons States of affairs are a simplest way to classify situations. For theoretical purposes, it is useful, though not strictly necessary, to extend our ontology with parameters. Parameters are constructs resembling the well-known operator of $[$-abstraction in the $[-$ calculus (Barendregt 1984). We start by adding some infinite set of parameters $\boldsymbol{a}_{1}, \boldsymbol{a}_{2}, \square$ for individuals, $\boldsymbol{r}_{1}, \boldsymbol{r}_{2}$, $\square$ 
for relations, etc. With these, we can now work with an abstract form of states of affairs, that we call infons. Infons are just like states of affairs, but some roles may be filled with parameters instead of concrete objects. The term 'infon' was coined by Keith Devlin (cf. Devlin 1991) to suggest that parametric states of affairs are theoretical entities that serve as the basic units of information. One can also think of infons as corresponding to properties. For example, the infon

$$
\square=<<\text { kisses, } \boldsymbol{a}_{1}, \boldsymbol{a}_{2} ; 1>>
$$

captures the property shared by all situations where someone is kissing someone.

The step from states of affairs to infons is a sort of abstraction. To get from the infons back to states of affairs, we need anchors. An anchor is a partial function from the domain of parameters to appropriate objects. We will always assume here that $f$ gives a value to all parameters involved. Where $f$ is an anchor and $<<\ldots$ $\boldsymbol{a}, \ldots>>$ is an infon, $\langle<\ldots \boldsymbol{a}, \ldots>>[f]=<<\ldots, f[\boldsymbol{a}], \ldots>>$. For instance, with $\square$ as above, we have $\square \boldsymbol{a}_{1} \square$ Anne, $\boldsymbol{a}_{2} \square$ Bill $]=<<$ kisses, Anne, Bill; $1>>$.

Notice that states of affairs are special infons: those whose set of parameters is empty. We can extend the earlier support relation I= to infons, by saying that an anchor $f$ satisfies an infon $\square$ relative to a situation $s$ if $s \mathrm{l}=\square[f]$. An anchor $f$ satisfies an infon $i$ simpliciter if $\mathrm{I}=\square[f]$, i.e., if there is a situation s such that $s$ $\mathrm{I}=\square[f]$. Finally, we say that $s \mathrm{l}=\square$ (s supports $\square$ ) if there is some anchor $f$ such that $s \mathrm{l}=[f f]$. For example, if $s$ is a situation where Anne is kissing Bill, then

$$
s \mathrm{l}=<<\text { kisses, Anne, } \boldsymbol{a}_{2} ; 1>>
$$

$$
\begin{gathered}
\text { because } s \mathrm{l}=<<\text { kisses, Anne, } \boldsymbol{a}_{2} ; 1>\left[\boldsymbol{a}_{2} \square \text { Bill }\right] \text {, so } \\
s \mathrm{l}=<<\text { kisses, Anne, Bill; } 1>>\text {. }
\end{gathered}
$$

Compound infons Infons so far are abstractions of single states of affairs. But there are natural ways to construct others. A guiding principle here is the Principle of Persistence: all information supported by a situation $s_{1}$ must be supported by any other situation $s_{2}$ that extends $s_{1}$. ${ }^{22}$ Two important constructs that comply with persistence are the meet of a set of infons and the existentialization of an infon with respect to a parameter. Where $\square$ is a set of infons, we can form the infon $\square \square$ such that an anchor $f$ satisfies $\square \square$ relative to situation $s$ if and only if $s$ $\mathrm{l}=\square[f]$ for all $\square$ in $\square$. As for existentials, given an infon $\square$ and a parameter $\boldsymbol{x}$, we

\footnotetext{
${ }^{22}$ This is like in intuitionistic logic, and partial logics: cf. the corresponding chapters in the Handbook of Philosophical Logic: van Dalen 2002, Blamey 2002.
} 
can construct the infon $\square x \square$ such that an anchor $f$ satisfies $\square x \square$ relative to situation $s$ if and only if for some object $a$, we have that $f_{x / a}$ satisfies $\square$ relative to $s$.

Of course, once we have constructs like these, we would like to have an algebra and logic of infons. We will not go in details here (see Devlin 1991, Moss \& Seligman 1997), but just point out that the usual logical intuitions cannot be taken for granted here, due to the fact that information is fine grained: logically equivalent compound infons may still be different pieces of information.

Situation types Once we have infons, we can abstract over situation parameters ${ }^{23}$, and thus obtain a rich collection of abstract situation types, of the form

$$
T=\left[\begin{array}{ll}
\boldsymbol{s} \mid & \boldsymbol{s} \mid=\square]
\end{array}\right]
$$

where $s$ is a situation parameter and $\square$ the conditioning infon of the type, $\operatorname{cond}(T)$. This infon may be a state of affairs, in which case we call $T$ a nonparametric type. If $T$ is a parametric type, we refer to it just as a type, and sometimes write $T(p)$ to stress that its conditioning infon $\square$ uses parameters $\boldsymbol{p}$. If $f$ is an anchor with domain $\boldsymbol{p}, T[f]$ denotes the type determined by the state of affairs $\square[f]$. We will overload the notation $\mathrm{I}=$ and use $s \mathrm{l}=T$ to mean that situation $s$ is of type $T$. Notice that this happens precisely when there is an anchor such that $s \mathrm{l}=[\mathrm{f}]$.

Constraints Now we are ready to introduce the notions which are at heart of the situation theoretic account of information. The crucial idea is that reality is full of regularities, lawlike constraints between types of situations, and it is the existence of those regularities that makes it possible for one situation to carry information about another. Constraints correspond to natural phenomena such as natural laws of physics, social conventions like those in language and traffic signs, and many other kinds of dependence. Formally, a constraint is an infon

$<$ Involves, $T[p], T^{\prime} ; 1>>$.

This infon is factual if the following existence condition is satisfied:

for every anchor $f$ with domain $\boldsymbol{p}$, whenever $s$ is a situation of type $T[f]$, then there is a situation $s^{\prime}$ of type $T^{\prime}[f]$.

If SMOKE is the type of situations where there is smoke and FIRE the type of those where something is burning, then $<<$ Involves, SMOKE, FIRE; $1>>$ is factual.

\footnotetext{
${ }^{23}$ There is a collection of that kind of parameters, too.
} 
Simple constraints do not make the kind of connection explicit that exists between the constituents of the two types involved. For that, we need relative constraints, infons of the form

$$
<\text { Involves }_{R}, T[p], T^{\prime}, T^{\prime \prime}[q]>>.
$$

Such a relative constraint is factual if for every anchor $f$ with domain $\boldsymbol{p} \square \boldsymbol{q}$, if $s$, $s$ " are two situations of types $T[f]$ and $T$ " [f], respectively, then there is also a situation $s^{\prime}$ of type $T^{\prime}[f] .{ }^{24}$

\subsection{Information flow}

Next we come to the fundamental issue of how one situation can carry information about another, which may be far away in time or space? We present two ways of formalizing this claim. The first is given here, in terms of the above ontology. The second requires the theory of classifications and channels (Barwise and Seligman 1997), and we leave it for the next subsection.

Propositions In situation theory, the bearers of truth are propositions, nonlinguistic abstract objects that have absolute truth values. There are two kinds of these. Russellian propositions are characterized just by a type $T$, and they are true if there exists a situation of type T. Austinian propositions are claims of the form $s \mathrm{l}=T$, thus involving a type and a situation. ${ }^{25}$ This distinction was highlighted by Barwise and Etchemendy (cf. Barwise and Etchemendy 1987), where it plays a key role in the treatment of semantic paradoxes.

Propositions are not infons. Infons characterize situations; propositions are truth bearers. We assume that for each situation type and each situation there is an Austinian proposition true just in case the situation is of that type. For Russellian propositions, we shall assume that for each type, there is a proposition true just in case some situation is of that type. This last strong assumption can lead to paradox (Barwise and Etchemendy 1987), but it will not affect us here.

Information Now, while propositions are the bearers of truth, it is particular situations that act as carriers of information. More precisely, it is the fact that some proposition $s \mathrm{l}=T$ is true, plus the existence of factual constraints relating $T$

\footnotetext{
${ }^{24}$ Intuitively, $T$ involves $T^{\prime}$ relative to $T^{\prime \prime}$ if for any pair of situations of the first and third types, there is a situation of the second type.

${ }^{25}$ If we adopted the controversial fourth thesis above that there is a total situation, then Russellian propositions could be taken as Austinian propositions determined by that total situation.
} 
with other types, that allows for $s$ to carry information about other parts of reality. The basic kind of informational statement for this reads:

the fact that $s \mid=T$ carries the information that Prop.

Depending on whether the proposition Prop is Russellian or Austinian, this tells us whether the information carried by $s$ is 'signal-bound' or 'incremental' (Israel and Perry 1990). In the Russellian case,

$s \mathrm{I}=T$ carries the signal-bound information that there is some situation of type $T^{\prime}$ if there is an anchor $f$ and a factual constraint $<<$ Involves $T_{1}, T_{2} ; 1>>$ such that $T_{l}[f]=T$ and $T_{2}[f]=T^{\prime}$.

If $s$ is a situation where there is an $x$-ray on the desk of a vet, we may say that

The $x$-ray's being $\square$-ish indicates that there is a dog, of whom this is an $x$-ray, and that dog has a broken leg.

This is a case of signal-bound information, since the information carried is about the signal itself (the $x$-ray). We can represent this regularity scenario as follows. First, the relevant factual constraint is $\mathrm{C}=<$ Involves, $T_{1}, T_{2} ; 1>$, where

$$
\begin{aligned}
& T_{1}=[s|s|=<<\{X \text {-ray, } \boldsymbol{x}, \boldsymbol{t}: 1>>\square<<\text { Has-pattern- } \square \boldsymbol{x}, \boldsymbol{t} ; \mathbf{1} 1>>] \\
& T_{2}=[s|s|=<<\text { Is- } X \text {-ray-of, } \boldsymbol{x}, \boldsymbol{y}, \boldsymbol{t} ; 1>>\square<<\text { Has-broken-leg, } \boldsymbol{y}, \boldsymbol{t} ; 1>>]
\end{aligned}
$$

The indicating situation $s$ will support the infon

$<<X$-ray, $a, t^{\prime} ; 1>><<$ Has-pattern- $\square, a, t^{\prime} ; 1>>$

where $a$ is the particular $x$-ray on the desk and $t^{\prime}$ is the time. This infon is an instantiation of $\operatorname{cond}\left(T_{1}\right)$ via the anchor $f(\boldsymbol{x})=a, f(\boldsymbol{t})=t^{\prime}$, so $s$ is of type $T_{l}[f]$. The signal-bound information carried by $s$ is the proposition that there is some situation of type $\mathrm{T}_{2}[f]$, that is, one that supports the infon

$<<$ Is- $X$-ray-of, $a, \boldsymbol{y}, t^{\prime} ; 1>>\square<<$ Has-broken-leg, $\boldsymbol{y}, t^{\prime} ; 1>>$

This says there is a $\operatorname{dog}$ with a leg depicted by $a$ at $t^{\prime}$ and that leg is broken at $t^{\prime}$.

But to guide a vet's action appropriately, it is not enough for her to be acquainted with the fact that the $x$-ray's is $\square$-ish and to be aware of the relevant constraint. She must also know that the information the $x$-ray carries is about Gretchen - i.e., the incremental information the $x$-ray carries, given the additional fact that it was taken of the specific dog Gretchen. We say that

the fact that $s \quad \mid=T$ carries the incremental information that $s^{\prime} \mid=T^{\prime}$ (relative to $T$ ") if there is an anchor $f$ and a factual relative constraint 
$<<$ Involves $_{R}, T_{1}, T_{2}, T_{3} ; 1>>$ such that $T_{1}[f]=T, T_{2}[f]=T^{\prime}$, and $T_{3}[f]=T^{\prime}$.

Notice that the informational content in this case is the Austinian proposition that $s^{\prime}$ I $=T^{\prime}$. Incremental information therefore, is information about a concrete situation $s^{\prime}$ via the indicating fact $s \mid=T$, in virtue of how $s$ is connected to $s^{\prime}$.

Our example turns on the relative constraint that, if an $x$-ray is of this type, and it is of a dog, then that dog had a broken leg at the time the $x$-ray was taken. That the $x$-ray was of Gretchen is the connecting fact, and the incremental information content is the proposition that Gretchen has a broken leg. The latter is about Gretchen, but not about the $x$-ray. The relevant factual relative constraint would be:

$$
\mathrm{C}^{\prime}=<<\text { Involves }_{\mathrm{R}}, T_{1}, T_{2}, T_{3} ; 1>>
$$

with indicating type $T_{1}$ as before, and indicated and connecting types $T_{2}$ and $T_{3}$ :

$$
\begin{aligned}
& T_{2}=[s|s|=<<\text { Has-broken-leg, } \boldsymbol{y}, \boldsymbol{t} ; 1>>] \\
& T_{3}=[s|s|=<<\text { Is- } X \text {-ray-of, } \boldsymbol{x}, \boldsymbol{y}, \boldsymbol{t} ; 1>>]
\end{aligned}
$$

As before, we assume that the indicating situation $s$ supports the state of affairs

$$
\square=<<X \text {-ray, } a, t^{\prime} ; 1>>\square<<\text { Has-pattern- } \square ; a, t^{\prime} ; 1>>
$$

Further, we assume that the connecting state of affairs $\square^{\prime}$ is factual. Where $b$ stands for Gretchen, $\square$ ' is $<<$ Is- $X$-ray-of, $a, b, t^{\prime} ; 1>>$.

Any anchor $f$ of the right kind here, with $\square=\operatorname{cond}\left(T_{1}\right)[f]$ and $\square^{\prime}=\operatorname{cond}\left(T_{2}\right)$, must be defined on the parameter $y$ of the connecting type: in particular, it must anchor $y$ to Gretchen. For any such anchor $f$, the proposition carried incrementally by the fact $s=T_{1}$ (relative to $\square$ ') states that there is a situation $s^{\prime \prime}$ with $s^{\prime \prime} \quad \mid=<<$ Hasbroken-leg, $b, t^{\prime} ; 1>>$. This is a proposition about Gretchen, not at all about the $x$ ray. And it is, after all, our real dog Gretchen that we are concerned about.

Agents using information Typically, information is used by agents to guide their actions, and thus, it is relative to the manner in which an agent is adapted to the world. This means the information an agent can get out of a situation depends to the constraints to which it is attuned. With the $x$-ray, what information is available to the vet depends on the regularities about $x$-rays to which she is attuned. We will now proceed to make this next set of notions more precise.

\subsection{Information flow and distributed systems}

So far, we have shown how the machinery of situation theory models the fact that one situation can carry information about another. It is easy to find examples of 
this flow beyond those already discussed. The manner in which the diaphragm of a microphone is vibrating carries information about what the announcer is saying. The modulation of the electromagnetic signal arriving at some antenna carries information about the way in which that diaphragm is vibrating. And finally, the modulation of the electromagnetic signal arriving at the antenna carries information about what the announcer is saying, for instance, "Hillary Clinton is irritated".

Situation theory provides tools for answering to the question: how does the modulation of the electromagnetic signal at the antenna carry information about the words that the announcer spoke? The theory relies on some fundamental principles about the nature of information as it flows across distributed systems, that is, systems that can be analyzed in sub-parts:

(1) The availability of information depends on the existence of regularities between connected parts of a distributed system.

(2) These regularities are relative to how the system is analyzed into parts.

(3) Flow of information crucially involves types and their concrete instances. It is in virtue of constraints (relations on types) that concrete situations, being of certain type, can act as carriers of (concrete or general) information.

We will now present a second, more mathematical way of formalizing the idea of one situation carrying information about another. This is done via the theory of classifications and channels (Barwise \& Seligman 1997). This paradigm, based on category theory, is an elegant formal distillation of the ideas presented so far.

\subsection{Classifications and channel theory}

The notions of information flow presented in the previous section involve facts of the form $s \mathrm{I}=T$, where $s$ is a situation and $T$ a type. These facts are about how situations are classified. The notion of classification, independently discovered around 1990 as 'Chu Spaces' (Gupta 1994) and 'Formal Contexts' (Ganter \& Wille 1997), is the basic notion on which the theory of classifications and channels is built. Classifications are triples, often depicted as follows:

$$
\prod_{\square}^{\square_{\square}}{ }^{\mathrm{I}={ }_{A}}
$$

Here $\square_{\square}$ is a set of tokens (for example, situations), $\square_{\square}$ is a set of types (conceived of as anything that can classify tokens), and $\mathrm{I}=$ is a relation between tokens and types. If $s$ is a token and $T$ a type, then $s \mid={ }_{\mathrm{A}} T$ reads as ' $s$ is of type $T$ '. 
The natural 'part-of' relationships that exist between parts of a system are called infomorphisms. An infomorphism $f: \boldsymbol{A} \square \boldsymbol{C}$ between two classifications is a pair $<f^{\natural}, f>$ of functions

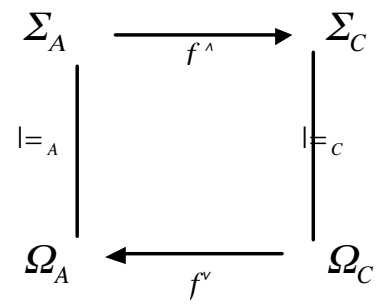

such that for all tokens $b \square \square_{C}$ and all types $T \square \square_{A}$

$$
f(c) \mid={ }_{\mathrm{A}} T \text { if and only if } c \mid={ }_{C} f^{\square}(T)
$$

Infomorphisms are of independent interest as an abstract invariance behind translation between theories, and that of general category-theoretic adjunctions. But here we look at their concrete uses as an intuitive model for information flow.

A concrete scenario The Smoke-on-the-Mountain scenario of Indian logic, mentioned in Section 1 of this chapter, involves at least two classifications $\boldsymbol{A}$ and $\boldsymbol{C}$. Tokens of $\boldsymbol{A}, \square_{A}$ might be situations where somebody is facing a mountain, while the relevant types $\square_{A}$ might include SEESSMOKE, LOOKINGUP, LOOKINGDOwN, BLIND etc. On the other hand, the classification $\boldsymbol{C}$ might correspond to the overall setting including the observer and the mountain. Its tokens are situations extending those of $\square_{A}$, and types in $\square_{C}$ might include OBSERVERSEESSMOKE, THEREISAFIREONTOP, etc. The map $f$ maps each large situation to the sub-situation capturing just the point of view of the observer. The map $f^{\natural}$ sends SMOKEOBSERVED to OBSERVERSEESSMOKE, LOOKINGDOWN to OBSERVERLOOKINGDOWN, etc. Thus type $\boldsymbol{T}$ of $\boldsymbol{A}$ is mapped to a type of $\boldsymbol{C}$ intended to mean that 'the observing situation' is of type $T$. Condition (*) ensures that things work out just this way.

As before, it is the existence of constraints, in the form of regularities between types that makes information flow within a distributed system. In this more general abstract setting, a constraint $\square_{1} \mid \square \square_{2}$ of classification $\boldsymbol{A}$ consists of two sets of types such that for all $a$ in $\square_{\square}$, if $a \mid==_{A} \square \square_{1}$, then $a \mid={ }_{A} \square_{2}$. If $\boldsymbol{A}$ were the classification of observers facing a mountain, SEESSMOKE, BLIND $\mid \square \varnothing$ would be a constraint of $\boldsymbol{A}$, saying that no blind observer sees smoke on top of the mountain.

Channels and information flow Let us now add to our observer and his mountain a third classification $\boldsymbol{B}$ for what is happening at the mountain top. Its tokens are situations located on mountain tops, and its types include things such 
as THEREISFIRE, THEREISFOG, etc. $\boldsymbol{B}$ is also a 'part' of the big component $\boldsymbol{C}$ - say, via the infomorphism $g$ depicted here:

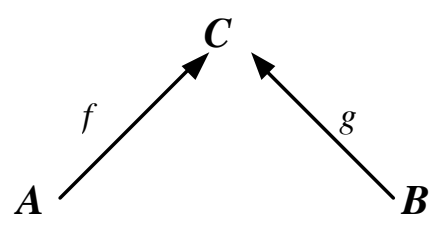

A collection of infomorphisms sharing codomain $C$ is called a channel with core $C$. Tokens of the core are called connections, because they connect subparts into a whole. Tokens $a$ from $\square_{A}$ and $b$ from $\square_{B}$ are connected in channel $\boldsymbol{C}$ if there is a token $c \square \square_{C}$ such that $f(c)=a$ and $g(c)=b$. In the example, an observing situation and a mountain top are connected if they belong to the same overall situation. We can now formulate a notion of (incremental!) information flow:

$a \mid={ }_{\mathrm{A}} T$ carries the information that $b \mathrm{l}{ }_{B} T^{\prime}$ (relative to $C$ ) if $a, b$ are connected in $C$ and $f^{\square}(T) \mid-g^{\square}(T)$ is a constraint of $C$.

This notion of information flow is relative to a channel -and hence, to an analysis of a whole distributed system into its parts. Again we see that 'carrying information' is not an absolute property: the mere fact that a token or situation is of a certain type does not completely determine what information it carries. ${ }^{26}$

Here is our observer-mountain example in these terms. Let $s \square \mathrm{A}$ be a situation of type SEESSMOKE: the observer in it sees smoke on top of the mountain. Let $s^{\prime} \square \mathrm{B}$ be the top-of-the mountain observed from the base of the mountain. Our choice of $\mathbf{C}$ makes $s$ and $s^{\prime}$ be linked by some connection in $\mathbf{C}$. In addition,

$$
\begin{aligned}
& f^{\natural}(\text { SEESSMOKE })=\text { OBSERVERSEESSMOKE } \\
& g^{\square}(\text { THEREISFIRE })=\text { THEREISAFIREONTOP. }
\end{aligned}
$$

Since OBSERVERSEESSMOKE ${ }^{\circ}$ THEREISAFIREONTOP is a constraint of $\mathbf{C}, s$ being of type SEESSMOKE carries the information that $s$ is of type THEREISFIRE.

Xerox Principle revisited The current information flow complies with Dretske's Xerox Principle, in the sense that we can compose channels. If we have two channels with cores $\boldsymbol{C}_{1}$ and $\boldsymbol{C}_{2}$, as those shown in the lower part of this diagram:

\footnotetext{
${ }^{26}$ This feature links up with general context dependency in logic (cf. Section 5).
} 


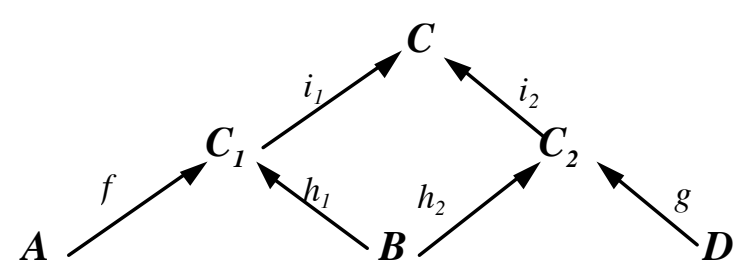

then we can always complete the diagram by taking the classification $\boldsymbol{C}$ to be the one whose tokens are ordered pairs of tokens of $\boldsymbol{C}_{1}$ and $\boldsymbol{C}_{2}$ and whose types belong to the disjoint union of those of $\boldsymbol{C}_{1}$ and $\boldsymbol{C}_{2}$. Here the type component of infomorphisms $i_{1}$ and $i_{2}$ is the identity, and tokens go via left and right projections. Within this setting, if $a \mid=_{A} T$ carries the information that $b \mid=_{B} T^{\prime}$ (relative to $C_{1}$ ) and $b \mid={ }_{B} T^{\prime}$ carries the information that $d \mid={ }_{D} T^{\prime \prime}$ (relative to $C_{2}$ ), then $a \mid=_{A} T$ carries the information that $d I={ }_{D} T^{\prime \prime}$ relative to the channel with core $C$ and infomorphisms $i_{1} f: \boldsymbol{A} \square \boldsymbol{C}$ and $i_{2} g: \boldsymbol{D} \square \boldsymbol{C}$.

Digression: model-theoretic relations between situations So far, we just assumed that a given distributed system has dependencies. But why does one situation correlate with another? Sometimes, this is a mere accident, just as funds in the stock market may have fluke correlations. But often, there are more intimate links. A watch keeps time as its stepwise operation mirrors the unfolding of time. Infomorphism is one elegant mathematical notion supporting such harmony. Following Barwise \& Seligman 1997, van Benthem 2000 has studied them model-theoretically, determining the precise first-order properties of structures which they preserve. But other model-theoretic relations between situations make sense, too, such as model extension to larger situations. For a study of modelcrossing notions of inference and logics of model change, cf. van Benthem 2007B. Following earlier work by Lindström, Barwise \& van Benthem 1999 study general 'information links', and entailment along model-crossing relations.

Local constraints and reasoning As we have seen, an agent's ability to get information from one part of a system based on what it can observe at another, depends on what constraints she is attuned to. Now, most regularities to which agents are attuned hold only within some regions of reality. If a massive object is released in the air, it will fall, given that we are on Earth, not in a satellite. The notion of local constraint captures this idea. A local constraint of classification $A$ has the form $\square \mathrm{I}_{\mathrm{A}} \square$ (on $S$ ), where $S$ is a collection of tokens from $A$, while $\square, \square$ are two sets of types from $A$, and for all $a \square \mathrm{S}$, if $a \mid=_{A} \square \square$, then $a \mid=_{A}$

A this point, it becomes natural to considering explicit constraint-based reasoning. Now in general, modeling agents that can reason about their world 
would require a good account of 'inferential information', which is not an easy notion to capture in a satisfactory manner (cf. Sections 7, 8 below). For instance, Barwise \& Seligman 1997 propose the following, following Dretske:

Inferential information: To an agent with prior knowledge $k, r \mathrm{I}=F$ carries the information that $s \mathrm{l}=G$ if and only if the agent can legitimately infer that $s \mathrm{l}=G$ from $r \mid=F$ and $k$, but not from $k$ alone.

But what does 'legitimately infer' mean in this setting? So far this question has not been satisfactorily answered. However, the notion of a local constraint has suggested some first approaches, which we survey here, following Martinez 2004. For a start, our setting validates local versions of basic inference rules, such as

Weakening

$$
\frac{\square_{1} \mathrm{I}_{A} \square_{2} \quad(\text { on } S)}{\square_{1}, \square_{1} \mathrm{I}_{A} \square_{2}, \square_{2}(\text { on } S)}
$$

Adding to the rule of Weakening all obvious identity axioms plus a strong form of the classical Cut rule, one can prove an abstract completeness theorem: If we fix $S$ and a collection of constraints on $S$, then the collection of theorems is precisely the theory (i. e., the set of all the constraints) of some classification.

But simple 'localization' of the usual rules does not allow for inferences where knowledge about one part of the system drives inferences about another. To achieve that, we need rules for shifting classifications (changing from $\mathrm{I}-{ }_{A}$ to $\mathrm{I}-{ }_{B}$ ) and conditions (from $S$ to another $S$ '). Martinez 2004 has rules for this, including:

S-Weakening

$$
\frac{\square_{1} \mathrm{I}-{ }_{A} \square_{2} \quad(\text { on } S)}{\square_{1}, \square_{1} \mathrm{I}{ }_{A} \square_{2}, \square_{2} \quad(\text { on } S} \square
$$

Enlargement

$$
\frac{\square_{1}, \mathrm{~T} \mathrm{I-}{ }_{A} \square_{2} \quad(\text { on } N e c(T, S))}{\square_{1}, \mathrm{~T} \mathrm{I-}{ }_{A} \square_{2} \quad(\text { on } S)}
$$

where $N e c$ is a function mapping each pair (type $T$, set of tokens $S$ ) to a subset of $S$ that includes all tokens of type $T$ in $S$. Natural modifications of other rules from Barwise \& Seligman 1997 would also yield principles such as

f-Intro:

$$
\frac{\square \quad \mathrm{I}_{A} \square(\text { on } S)}{f[\square] \mathrm{I}_{C} f[\square]\left(\text { on }(f)^{-1}[S]\right)}
$$

where $f[\square]$ is the set of types obtained by applying $f^{\square}$ to all types in $\square$. It is still an open question if calculi based on these rules are enough to formalize the notion of 'legitimate inference' in this setting in a fully satisfactory manner. 


\subsection{Harnessing information}

How agents use the information available to them is crucial in cognitive modeling. An agent's ability to make inferences is just one aspect here. A more fundamental (and general) reason why agents are able to use information is structural: the architecture of the agent is geared towards doing so (Israel \& Perry 1991). This turn fits well with the general Tandem View in this chapter, found also in other approaches, that notions of information must eventually be understood in terms of the dynamic processes which that information is used for. Now to specifics!

Clearly, for information to be of use to some agent, or to enable a device to do what it is supposed to, it is not enough that the states of the agent or device carry the information. The agent or device must in some sense have the information. We conceive of an agent having information as meaning that the architecture of the agent 'harnesses' the information to trigger action that advances the agent's goals. This architecture can be due to Nature or to a designer, and the goal can be a natural goal of the agent, or a goal for which the designer creates the agent.

For simplicity, assume an agent with one available action $A$ and one goal $G$. Relative to these, circumstances can be divided into a range of possibilities, $P$ and $\neg P$, those in which the action achieves the goal, and those in which it does not. ${ }^{27}$ The circumstances in $P$ are the success conditions of $A$ relative to $G$. The idea of harnessing information is now simple: some state of the agent that carries the information that $P$ should trigger the action $A .{ }^{28}$ An old-fashioned mousetrap provides a simple example of these ideas.:

Cheese is placed on a small lever. The trap is placed somewhere, behind the refrigerator say, where the only likely cause of the lever moving is the presence of a mouse directly in front of it. The trap has one action: a wire-blade is released. The goal is killing a mouse; this will occur when there is a mouse in the path of the blade. The trap is constructed so that a mouse causing the lever to move will be in the path of the blade, and the movement of the lever will release the blade.

\footnotetext{
${ }^{27}$ This is, by the way, the standard story from Decision Theory, except that we are ignoring risk or uncertainty with respect to the state of the reality.

${ }^{28}$ Here is a more general way of thinking about this. Any event will carry all sorts of information, relative to different constraints and connecting facts. It is the range of possibilities determined by the goals and actions that determines the information that is relevant. This again links information as range - now basically as having information about success conditions and information as correlation - carrying information about the world external to the agents.
} 
What we want then is an account of having information as being in a state that plays two roles (Israel \& Perry 1991). First, the agent's being in the state carries certain information relative to a constraint. Second, an agent's being in that state has an effect, i.e., it triggers an action relative to some other constraint, that is appropriate given the information. In that case, the agent not only carries but has the information. More elaborate formalizations of the relevant events occurring in such scenarios might involve a mixture of ideas from situation theory and dynamic logic - witness the brief discussion in Section 6.5 below.

\subsection{Some further developments}

Circularity and set theory Situation theory has been applied to information flow involving self-reference. Natural language as a medium for communication sometimes involves utterances about the very same situation in which they are made. Now, in set-theoretic approaches to situation theory, situations are usually modeled as sets of tuples (standing for the infons supported by the situation), but self-referring situations cannot be naturally modeled as sets in the standard Zermelo universe. This is because the Axiom of Foundation bans the existence of chains of sets of the form $u \quad u_{1} \square \square \square u$. Thus, a theory of sets without Foundation would fit situation theory better. Indeed, the study of circularity and its applications has been an important contribution of situation theory.

Technically, most of this work has taken place in $A F A$ set theory, which consists of $Z F C$ with the Foundation Axiom replaced by this Antifoundation Axiom (cf. Forti \& Honsell 1983, Aczel 1988, Barwise \& Moss 1996):

Given any graph $(W, R)$ whose childless nodes are labeled with urelements, there is a unique way to label the other nodes with sets such that, if $d(v)$ is the label of $v$, then $d(v)=\{d(u) \mid v R u\}$ (i.e., $d(v)$ is the set of labels of v's children).

A graph with its childless nodes labeled by urelements represents a set, and each set can be represented as a labeled graph. In fact, the same set may be represented by different, but bisimilar labeled graphs. ${ }^{29}$ E.g., there is a unique set with the self-referential or circular specification $\square=\{\square\}$, and it can be represented by the following two graphs (among many other bisimilar ones):

\footnotetext{
${ }^{29}$ We already encountered bisimulation in a different guise in Section 3, as a measure of identity for information structure in epistemic logic.
} 


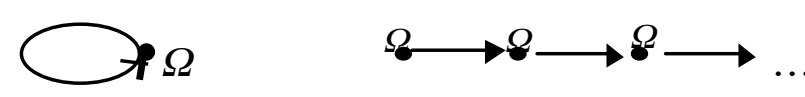

Bisimilarity enters the picture here, because in the $A F A$ universe, equality of elements is not enough as a criterion for deciding whether two sets are equal.

Circularity, modal logic, and co-algebra The preceding facts lead to interesting connections with modal logic and the use of recent co-algebraic methods. ${ }^{30}$ Given a monotone operator $F$ on sets, an $F$-coalgebra is just a mapping $f: X \square$ $F(X)$. As a simple but important example, when we take $F(X)=P(X)$, an $F$ coalgebra turns out to be just a modal relational Kripke frame. Main themes in this new direction are the duality between algebras, inductive definitions and smallest fixed points on one hand, and coalgebras, coinductive definitions and largest fixed points on the other hand (Jacobs \& Rutten 1997). For the modal logic connection, see Venema 2006. Barwise \& Moss 1996 is a pioneering study of the general theory, starting from work by Aczel, with a mixture of $A F A$ set theory and early co-algebra, and Baltag 1999 is a further study of $A F A$-classes. ${ }^{31}$

Summary and further applications Situation theory is a logical account of information in distributed systems (including reality itself as parsed by agents) which model information as correlation. It brings along its own agenda of technical issues, which include formal theories of circularity in modeling and the associated reasoning apparatus, and a general account of relations between situations and of the channels along which information can flow. For further applications, we refer to the more linguistic/social-science chapter by Devlin \& Rozenberg in this Handbook, as well as more computational studies such as Akman \& Tin 1997 and Martinez 2004. Finally, Devlin 1991 is a good introduction to the general ideas and state of the art in the early 1990s.

\section{$6 \quad$ Merging Range and Constraint Views}

Sections 3 and 5 have told two separate logical semantic stories of information, once as range and once as correlation. In this section, we compare the two, and

\footnotetext{
${ }^{30} \mathrm{Cf}$. SanGiorgi 2007 for an excellent history of the history of the notion of bisimulation since the 1970s, through modal logic, theories of computation, and set theory.

${ }^{31}$ This last issue has been important beyond purely technical reasons. Although the initial intuition about situations sees them as 'small', situation theory actually deals with large set-theoretic objects. For instance, the treatment of paradoxes via Austinian propositions allows for models of reality resolving all possible issues, which turn out to be proper classes (Barwise \& Etchemendy 1987).
} 
propose some merges, in line with parts of the computational literature. While we cannot do justice to all aspects of this comparison here, we do show that the two styles of thinking are very congenial.

Many readers might rather have expected a polemical shoot-out here. It is often thought that epistemic logic and situation theory are hostile paradigms, witness discussions between, e.g., Perry and Stalnaker in the 1980s. For instance, much has been made of the partiality of situations versus the 'totality' of possible worlds. But in practice, many of these differences are minor. In many models of epistemic logic, possible worlds are small and partial. In the card examples in Section 2, 'worlds' are just possible hands, and hence 'no big deal'. Conversely, some entities cited as 'situations' can be pretty complex, such as countries, or world-wars. Also, situation theory has been cast as 'realist' and non-modal, in that only chunks of the real world are needed in its semantics. But this difference, too, evaporates, once one tries to give a situation-theoretic account of belief and being wrong (cf. Kratzer 2006). Significantly, Barwise 1997 eventually introduces some sort of possible worlds and modal languages into situation theory. Indeed, the more striking historical development has been the steady discovery of significant analogies between situation theory and modal logic. This has happened to such an extent that, in the definitive mathematical framework of Barwise \& Moss 1996, modal logic has become the vehicle for developing a bisimulation-based situation theory! Indeed, the two paradigms seem compatible and congenial both technically and conceptually - and they share a common cause.

This section emphasizes three themes, following van Benthem 2005B:

(a) modal and first-order logics of dependence as an account of constraints,

(b) merges between constraint-based models and dynamic epistemic logic,

(c) the addition of events to create dynamic versions of situation theory.

This topic is more a digression in this chapter, but it serves two purposes. First, since both aspects of information are relevant in practice, we need to understand how epistemic logic and situation theory can live together in modeling actual phenomena. Moreover, juxtaposing these systems raises some interesting new questions, and allows us to see where both lie in the larger picture of logic today.

\subsection{Correlation through gaps in state spaces}

In a world of one-shot events, no significant information can flow. Genuine constraints only arise in situations with different 'states' that can be correlated. To make this more precise, consider two situations $s_{1}, s_{2}$, where $s_{1}$ can have some 
proposition letter $p$ either true or false, and $s_{2}$ a proposition letter $q$. There are four possible configurations:

$$
\begin{array}{|ll}
s_{1}: p, s_{2}: q & s_{1}: p, s_{2}: \neg q \\
s_{1}: \neg p, s_{2}: q & s_{1}: \neg p, s_{2}: \neg q
\end{array}
$$

With all these present, one situation does not carry information about another, as $p$ and $q$ do not correlate in any way. A significant constraint on the total system arises only when we leave out some possible configurations. For instance, let the system have just the following two states:

$$
s_{1}: p, s_{2}: q, \quad s_{1}: \neg p, s_{2}: \neg q
$$

Now, the truth value of $p$ in $s_{1}$ will determine that of $q$ in $s_{2}$, and vice versa. In a formula with some obvious notation, we have the truth of the following constraint:

$$
s_{1}: p \square s_{2}: q
$$

But even a less constrained system with three instead of just two global configurations still allows for significant information flow:

$$
s_{1}: p, s_{2}: q, \quad s_{1}: \neg p, s_{2}: q, \quad s_{1}: \neg p, s_{2}: \neg q
$$

Presence of $p$ in $s_{1}$ still conveys the information that $q$ in $s_{2}$, but absence of $p$ does not convey information about $s_{2}$. Again in a formula, we have the implication:

$$
s_{1}: p \square s_{2}: q
$$

Contraposing the implication, absence of $q$ in $s_{2}$ tells us about absence of $p$ in $s_{1}$, but presence of $q$ has no immediate informative value about $s_{1}$.

Thus, correlation between different situations amounts to restrictions on the total state space of possible simultaneous behaviors. The more 'gaps' in that state space, the more information there is in the system, to be used by potential observers. ${ }^{32}$ The bare bones of this setting are brought out by constraint models

$$
\boldsymbol{M}=(\text { Sit }, \text { State }, \boldsymbol{C}, \text { Pred })
$$

\footnotetext{
${ }^{32}$ Recall that in epistemic logic, gaps in the set of worlds encode common knowledge.
} 
with a set Sit of situations, a set State of possible valuations, a predicate Pred recording which atomic predicates hold where, and crucially, a 'constraint relation' $C$ stating which assignments of states to situations are possible in the system. ${ }^{33}$

\subsection{Modal logics of constraints and correlation}

Constraint models suggest a logic - in the simplest case, a modal one. To see this, take a language with names $x$ for situations (a tuple $x$ names a tuple of situations), and atomic assertions $P \boldsymbol{x}$ for properties of or relations between situations. We take Boolean operations, plus a universal modality $U \square($ ' $\square$ is true everywhere'):

$$
P x|\neg| \quad \mid U
$$

The semantic interpretation has obvious clauses for the following notion:

$$
\boldsymbol{M}, \boldsymbol{s} \mid=\square \quad \square \text { is true in global state } \boldsymbol{s} \text { of model } \boldsymbol{M}
$$

In particular, $P \boldsymbol{x}$ holds at $\boldsymbol{s}$ if the tuple of local states assigned by $\boldsymbol{s}$ to the tuple $\boldsymbol{x}$ satisfies the predicate denoted by $P$. This language defines basic constraints across situations such as $U(P x \square Q y)$. The resulting logic is classical propositional logic plus the modal logic $S 5$ for the universal modality $U$.

But we can go one step further. Intuitively, a situation $x$ which satisfies $p$ 'settles' the truth of $p$, plus all repercussions this has for other situations in the system. Accordingly, define the following new relation between global states:

$$
\boldsymbol{s} \sim_{x} \boldsymbol{t} \text { iff } \boldsymbol{s}(x)=\boldsymbol{t}(x) .
$$

This generalizes to a relation $\sim_{x}$ for sets or tuples of situations $x$ by requiring equality of $\boldsymbol{s}$ and $\boldsymbol{t}$ for all coordinates in $\boldsymbol{x}$. Thus, there are modalities []$_{x} \square$ for each such tuple, which say intuitively that the situations in $x$ settle the truth of $\square$ in the current system:

$$
\boldsymbol{M}, \boldsymbol{s} \mid=[]_{x} \square \quad \text { iff } \quad \boldsymbol{M}, \boldsymbol{t} \mid=\square \text { for each global state } \boldsymbol{t} \sim_{x} \boldsymbol{s}
$$

This language can express more subtle properties of information.

Digression One vivid metaphor here views situations themselves as agents. Operators []$_{x},[]_{x}$ then express what single situations or groups know by inspecting their own local properties. This epistemic interpretation of constraint

\footnotetext{
${ }^{33}$ We are not claiming any originality for this set-up. Constraint models are like 'context models' or 'local semantics' in the style of Ghidini \& Giunchiglia 2001, and they also resemble the local state models of 'interpreted systems' in the style of Fagin et al. 1995 - be it with a non-epistemic interpretation, for now.
} 
models is strengthened by one more analogy. The tuple modalities []$_{x}$ involve an intersection of accessibility relations []$_{x}$ for single situations $x$. This is like 'distributed knowledge' for groups in epistemic logic, describing what whole sets of agents may be said to 'know implicitly'. ${ }^{34}$

As to valid laws, constraint models satisfy persistence properties for atomic facts:

$$
P x \square[]_{x} P x, \quad \neg P x \square[]_{x} \neg P x \quad 35
$$

More generally, the extended modal constraint language has a decidable complete logic with modal $S 5$ for each tuple modality, plus all axioms of the forms $U \square \square$ []$_{x} \square$, and []$_{x} \square \square \quad[]_{y} \square$ whenever $\boldsymbol{y} \square \boldsymbol{x}$.

\subsection{Digression: constraint logic and logic of dependence ${ }^{36}$}

A natural alternative to our story of situations and constraints involves another major notion in current logical theory, viz. dependence. Dependence fits well with information as correlation, and as we shall see, also with information as range. To bring this out, we can stay close to standard first-order logic.

For a start, think of the earlier situations as variables $x, y, \ldots$ which store values. A global state $s$ is then a variable assignment in the usual sense: a function assigning an object to each variable. Now first-order logic has no genuine dependencies between variables. In any assignment $s$, we can shift the value of $x$ to some object $d$ to obtain a new assignment $s[x:=d]$, where all other variables have retained their $s$-value. This is the reason why first-order logic typically has validities like the following commutation of quantifiers:

\section{$\square x \square y \square \square \square y \square x \square$}

The order of assigning values to the variables $x$ and $y$ is completely independent. But in many natural forms of reasoning, e.g., in probability theory, variables $x, y$ can be dependent, in the sense that changes of value for one must co-occur with changes of value for the other. Such cases of genuine dependence can be modeled in a first-order setting (van Benthem 1996, Chapters 9, 10). A general assignment

\footnotetext{
${ }^{34}$ Similar points have been made by Baltag \& Smets in their dynamic logic analysis of information structure and measurement-driven information flow in quantum mechanics.

35 These implications do not hold for all formulas. E.g., $\square_{x} P x \square \square_{y} \square_{x} P x$ is invalid, since accessible global states for $x$ may change after a shift in the $y$ coordinate.

${ }^{36}$ This section may be skipped without loss of continuity, but the main ideas are really simple, and they connect the preceding analysis with the heartland of logic.
} 
model is a pair $(\boldsymbol{M}, \mathbb{V})$ of a first-order model $\boldsymbol{M}$ with domain $D$ and interpretation function $I$, and $V$ a non-empty set of assignments on $\boldsymbol{M}$, i.e., a subset of the total space $D^{V A R}$. The first-order language is now interpreted as usual, but using triples $\boldsymbol{M}, \mathbb{V}, s$ with $s \square \mathbb{V}$ - with the following clause for quantifiers:

$M, \mathbb{V}, s \mid=\square x \square$ iff for some $t \square \mathbb{V}: s={ }_{x} t$ and $M, V, t \mid=\square$

Here $=x$ relates assignments identical up to $x$-values.

The analogy with the earlier constraint language will be clear. Moreover, in this broader semantic setting, we get new dependence operators, such as polyadic quantifiers $\square \boldsymbol{x}$ binding tuples of variables $\boldsymbol{x}$ :

$M, \mathbb{V}, s \mid=\square x \square$ iff for some $t \square \mathbb{V}: s={ }_{x}$ t and $M, \mathbb{V}, t \mid=\square^{37}$

In first-order logic, $\square x y \bullet \square$ is just short-hand for $\square x \square y \square$ or $\square y \square x \square$ in any order. But in general assignment models, these expressions are no longer equivalent, as not all 'intermediate assignments' for $x$ - or $y$-shifts need be present - and both fail to capture $\square x y \bullet$ as defined here. ${ }^{38}$

The complete logic of general assignment models is a decidable subsystem of standard predicate logic. It contains those valid first-order laws which hold even when variables may be correlated (Németi 1995, van Benthem 1996, 2005A). Beyond this decidable core logic, further axioms express special features of constraint models. E.g., the commutativity law $\square x \square y \square \square \square \square x \square$ says that a Diamond Property should hold:

If $s \sim_{x} t \sim_{y} u$, then there is another available assignment $v$ with $s \sim_{y} v \sim_{x} u$. Imposing such special conditions makes models much like full function spaces, and the complete first-order logic (of independence) becomes undecidable.

Van Benthem 2005 shows how the above modal constraint logic can be faithfully embedded into the first-order logic of dependent variables, and also vice versa. Thus, constraints and dependence are the same topic in two different guises! Dependence of various kinds is a major theme in foundations of first-order logic these days (Abramsky 2006, Väänänen 2007). We have shown this is also a move toward a logic of information and constraints in the situation-theoretic sense.

\footnotetext{
${ }^{37}$ Here, $=\boldsymbol{x}$ is identity between assignments up to values for all variables in $\boldsymbol{x}$.

${ }^{38}$ In these models, one can also interpret single or polyadic substitution operators in their own right: $\boldsymbol{M}, \mathbb{V}, s \mathrm{I}=[\boldsymbol{y} / \boldsymbol{x}] \square$ iff $\mathrm{s}[\boldsymbol{x}:=s(\boldsymbol{y})] \square \mathbb{V} \& \boldsymbol{M}, \mathbb{V}, s[\boldsymbol{x}:=s(\boldsymbol{y})] \mathrm{I}=\square$.
} 


\subsection{Combining epistemic logic and constraint logic}

Intuitively, information as correlation and information as range seem different notions. The difference is one of agency. A blinking dot on my radar screen carries information about some airplane approaching. But it does so whether or not I observe it. I may 'have' the information about the airplane, when I am in a situation at the screen, but unless I know that there is a blinking dot, it will not do me much good. That knowledge arises from an additional event, my observing the screen. If I make that observation $S$, and I know the right constraint $S \square A$, I will indeed also know that there is an airplane $A$.

Though distinct, the two phenomena obviously form a natural and compatible pair. To bring them together technically in a simple setting, we can use a combined modal logic of constraints and knowledge, first static and eventually also dynamic. We give one system, just to show how easy this is, viz. a combined epistemic constraint language with syntax

$$
P \boldsymbol{x}|\neg| \quad|U|[]_{x} \mid K_{i}
$$

Epistemic constraint models are then bi-modal structures of the form

$$
\boldsymbol{M}=\left(\text { Sit }, \text { State }, \boldsymbol{C}, \text { Pred }, \sim_{i}\right)
$$

where global states have the earlier component-wise relations modeling constraints, while there are additional abstract epistemic accessibility relations $\sim_{i}$ for each agent $i$. Specifics of the latter depend on how the relevant scenario specifies agents' access to the situational structure. ${ }^{39}$ We now have a simple language combining correlation and range talk. E.g., suppose that our model $\boldsymbol{M}$ satisfies the constraint $s_{1}: p \square s_{2}: q$. Then the agent knows this, as the implication is true in all worlds in $\boldsymbol{M}$. Now suppose the agent knows that $s_{1}: p$. In that case, the agent also knows that $s_{2}: q$, by the Distribution law of epistemic logic:

$$
\left(K s_{1}: p \square K\left(s_{1}: p \square s_{2}: q\right)\right) \square K s_{2}: q
$$

The converse requires more thought. The point is not that the agent already knows that $s_{1}: p$, but that, if she were to learn this fact, she would also know that $s_{2}: q$. In our earlier dynamic-epistemic terms, this would read as follows:

$$
\left[! s_{1}: p\right] K s_{2}: q
$$

\footnotetext{
${ }^{39}$ The general logic of epistemic constraint models is a mere fusion of that for constraint models and a poly-S5 epistemic logic of knowledge. In the case of one single agent, the earlier universal $U$ then serves as an epistemic $K$.
} 
This formula is equivalent to the truth of the constraint - by the axioms for dynamic-epistemic logic (cf. Section 4). Next, what do agents know about the informational content of specific situations $x$ ? If []$_{x} \square$ holds at some world $s$, must the agent know this fact: []$_{x} \square \square \square[]_{x} \square$ ? Not so: []$_{x} \square$ can be true at some worlds, and false at epistemically accessible ones. What a situation $x$ 'knows' in the earlier impersonal sense of correlation need not be known to an external agent, unless this agent makes an observation about $x$. Thus, a combined modal-epistemic logic brings out the interaction between our two senses of information.

Digression: interpreted systems and a uniform vector view In the paradigm of 'interpreted systems'(Fagin, et al. 1995), epistemic worlds are vectors of 'local states' for individual agents, and epistemic accessibility between worlds $s, \boldsymbol{t}$ for agent $i$ is just component-wise equality $(s)_{i}=(t)_{i}$. This structured view of possible worlds, extends to $\sim_{i}$ for groups of agents $i$, just as we did in constraint models with the move from $\sim_{x}$ to $\sim_{x}$. On such a view, epistemic accessibility for agents and constraint accessibility for situations as part of the relevant physical world become formally analogous. Accordingly, we can then also use one uniform vector format for our combined models. Consider once again the earlier example

$$
s_{1}: p, s_{2}: q \quad s_{1}: \neg p, s_{2}: \neg q \quad s_{1}: \neg p, s_{2}: \neg q
$$

Let some agent $i$ have an accessibility structure indicated by the black dotted line:

$$
s_{1}: p, s_{2}: q \cdot \square \cdot s_{1}: \neg p, s_{2}: \neg q \quad s_{1}: \neg p, s_{2}: \neg q
$$

We can bring this into vector format by casting the agent itself as a further component, which can be in one of two states, as in the following picture:

$$
\begin{gathered}
s_{1}: p, s_{2}: q, i: \text { state-1 } \quad s_{1}: \neg p, s_{2}: \neg q, i: \text { state- } 1 \\
s_{1}: \neg p, s_{2}: \neg q, i: \text { state }-2
\end{gathered}
$$

The component-wise accessibility is the same as in the preceding picture. ${ }^{40}$

To summarize, information as correlation and information as range co-exist happily inside one formal modeling, and that even in more than one way.

\footnotetext{
${ }^{40}$ Interpreted systems also involve stepwise action by agents, but more on this below.
} 


\subsection{Explicit dynamics: events, information change, and correlation}

But the compatibility extends to the dynamics of both. Dynamic epistemic logic made informational events a key feature. Likewise, situation theory involved event scenarios for making use of ('harnessing') information, such as The Mousetrap in Section 5. Indeed, the constraint models of this section have a dynamic aspect in the first place. Constraints relate different situations, and the most useful correlations (think of the ground station and the mountain top) involve events over time, leading to different global states of some evolving system.

It seems of interest to bring out this temporal dynamics explicitly in some logic. Many situation-theoretic scenarios may be viewed as specifying automata consisting of many parts which can undergo correlated changes, through actions or events $e$. The Mousetrap and other running examples are of this kind: a complex system with a finite number of components cycles through a finite number of possible states, according to some fixed scenario. This style of analysis may be represented in combined dynamic constraint models

$$
\boldsymbol{M}=(\text { Sit }, \text { State }, \boldsymbol{C}, \text { Pred }, \text { Event })
$$

where events $e$ are binary transition relations between global states. For instance, we may have had absence of a fire and a smoke signal, and then a combustion event takes place, changing the global state in our Mountain Top setting to (smoke, fire). The matching language combines the earlier constraint operators with dynamic event modalities:

$$
P \boldsymbol{x}|\neg||U|[]_{x} \mid[e]
$$

Here, we interpret the dynamic modality in the usual modal style:

$$
\boldsymbol{M}, \boldsymbol{s} \mathrm{I}=[e] \square \quad \text { iff } \quad \text { for all } \boldsymbol{t} \text { with } \boldsymbol{s} \boldsymbol{R}_{e} \boldsymbol{t}: \boldsymbol{M}, t \mid=\square
$$

This language describes constraints on the current state, but its event modalities also record what happens as the system moves in the space of all its possible developments. ${ }^{41}$ These merged logics seem easy to use and perspicuous. ${ }^{42}$

\footnotetext{
${ }^{41}$ A more realistic language would have local events at specific locations, making global events tuples of simultaneous local ones. This would be a form of 'concurrent' dynamic logic. ${ }^{42}$ A connection with DEL. Suppose that a system has 2 states, and we do not know if it is in $P$ or $\neg P$. Now we perform an observation, and learn that the state is $P$. This is not an internal 'system event', as it affects an outside agent's view of the system. In Section 4, public observations $! P$ changed epistemic models $\boldsymbol{M}$ to sub-models $\boldsymbol{M} \mid P$ with domain $P^{M}$. Both internal events and external observations can be implemented
} 
More sophisticated scenarios combining epistemic information, correlations, and informational events are discussed in Baltag \& Smets 2007 on the structure of quantum information states and measurement actions. Its state spaces seem quite close to the view presented in this section, but now in Hilbert spaces.

If we want to describe longer-term system properties describing fixed and perhaps even shifting correlations over time, an earlier richer option is available. Epistemic temporal languages for multi-component systems in the sense of Fagin et al. 1995, or Parikh \& Ramanujam 2003 describe the long-term behaviour of processes and agents over time, and they include intertwined accounts of external events and internal message passing. ${ }^{43}$ Van Benthem, Israel \& Perry 2007 discuss how to explicitly model the implicit dynamics in the core situationtheoretic scenarios for 'harnessing information' in dynamic and temporal logics.

\subsection{Conclusion: semantic co-existence}

Information as range gave rise to both static and dynamic epistemic logics. Likewise, information as correlation leads to static and dynamic constraint logics. The two can be merged in a modal setting, implementing the schema in Section 1, where systems change over time, adapting their connections to agents who are informed about them, and to the reality they are about. Thus information as range and as correlation are intimately related. The two agendas merge naturally into one logical semantic view of information structure and dynamics.

\section{Information as Code: Syntax, Proof and Computation}

\subsection{Information as code and elucidation}

Our analysis so far has been largely semantical in orientation, and it seems successful in fitting logical notions of information into one perspective. But as noted at the start of this chapter, logic has another broad take on information structure and its processing, viz. inference on formulas. The relevant focus is then syntax and proof theory rather than model theory. This is a vast area, and we only discuss a few basic issues here, fleshing out a few relevant features of what might be called information as code - with a matching dynamics of elucidation.

in dynamic constraint models by making agents components of the global state as before. The agent is trying to find out the current state $s$, but the language can also express the fact that $\boldsymbol{s}$ may change to $\boldsymbol{t}$ after system-internal events $e$.

${ }^{43}$ This also seems the proper setting for a logical account of the crucial issue of how our information and the beliefs based on it 'track' the world over time (cf. Roush 2006). 
For a start, the precise notion of 'information' behind the many calculi of inference in modern logic - and an optimal level of abstraction for it -, seems even less established than in logical semantics. Inference by its nature is more tied to details of syntactic representation, either on formulas per se, or some abstraction thereof. This allows for more variation in what counts as information states than our earlier semantic models. In line with this, there is a great variety of logical systems for deduction and proof, with very different formats. Standard mathematical Proof Theory (Troelstra \& Schwichtenberg 1996, Feferman 2000, Tennant 1978) uses natural deduction and associated calculi of type theory. Other well-established formats that have been linked with modeling information flow are logic programming and resolution theorem proving (cf. Kowalski 1979), Doets 1994), and more general unification-based inferential mechanisms (cf. Rounds 1997).

Proof theory is a rich field, and a whole chapter parallel to the present one might be written in its terms. Moreover, it has its own natural levels of abstraction for dealing with information in type theory or category theory, making room for a variety of logics: classical, intuitionistic, or sub-structural (Prawitz 1965, Belnap 1982, Restall 2000). We will not go into this here. Instead, we discuss a few general issues linking up with our earlier sections. We refer to the chapter by Abramsky in this Handbook for a state-of-the-art account of proof structure as it relates to the information flow in computation, another key process of elucidation.

\subsection{How can inference be informative?}

Let us start off with a somewhat surprising question, to which no definitive answer appears to be known. Simply put, our earlier semantic theories of information have the following problem. Semantic approaches do not account for the informative nature of inference! Depending on where one puts the blame, this problem may be formulated in various terms. Jaakko Hintikka has called it 'the scandal of deduction'. This is a real problem when we look at logical practice. For, deduction is clearly informative. Natural tasks show a clear interplay of update through observation, or recollection, or whatever source, with more combinatorial inferential steps. When solving a puzzle, we do not just update information spaces, we also make appropriate deductions which highlight some important aspect of the solution, or at least, the road toward it.

Example: update and/or inference In practice, reasoning often trumps update. Here is a simple toy example. You are throwing a party subject to the following constraints: (a) John comes if Mary or Ann does, (b) Ann comes if Mary does not, (c) If Ann comes, John does not. Is this a feasible event? In principle, one can 
update as in Section 2 here, starting with 8 possible sets of invitees, and then ruling out 3 by (a), 2 more by (b), and finally, using (c) to cut down to the only solution remaining. But in reality, you would probably do something more like this (with logicians' annotation):

By (c), if Ann comes, John does not. But by (a), if Ann comes, John does: a contradiction, so Ann does not come. Then, by (b), Mary comes. By (a) once more, John comes. Indeed, \{John, Mary\} satisfies all requirements.

There is a clear sense in which these successive inferential steps add relevant information. Indeed, cognitive science tells us (Knauff 2007) how in cognitive tasks, the brain continually mixes model inspection and inferential steps. But there is a difficulty in saying just how the latter process can be informative. An inference step does not shrink the current information range, and it does not add to correlational constraints. It rather adds information in a more combinatorial sense, which may depend on the agent's coded representations and even purposes. Other versions of this problem are known in the philosophy of science. E.g., much has been written about the way deductive consequences of a physical theory, such as Einstein's deduction for the perihelion perturbation of Mercury from the General Theory of Relativity (Fitelson 2006), can uncover startling new information which a scientific community did not have before.

Information in computation This problem is not unique to logic: witness several chapters of this Handbook. Inference is much like computation, and one issue running through the chapter by Abramsky in this Handbook is how computation can provide information, and how abstract semantics can provide a handle on this. In a more standard mathematical framework, the chapter by Adriaans analyzes information growth through computation in terms of numerical measures such as Kolmogorov complexity. There, too, inference or computation can increase information, but only by a little, since a shortest description of the theorem generator plus the allotted running length suffices. Thus, the divide between semantical and code-based approaches to information occurs outside of logic, too. In what follows we discuss a few strands at the syntax/semantics interface.

\subsection{Logical syntax as information structure}

In response to the challenge of information flow through deduction, various answers have been given. For instance, Hintikka 1973 proposed a way inside predicate logic for distinguishing levels of information in the syntactic analysis of 
a formula. First-order formulas $\square$ describe the existence of objects with certain properties while excluding others, and these enumerations of object types can be unpacked level by level until the full quantifier nesting depth of $\square$. Inferences that stay close to the original syntax contain only 'surface information', those requiring full processing into normal form carry 'depth information' - notions which have been elaborated in some numerical detail. ${ }^{44}{ }^{45}$ All this is clever, and it suggests that logical syntax might have natural information content where syntactic manipulation can increase information. But it is also very system-dependent. So far, no coherent and generally useful notion of syntax-based information has evolved - partly because we have no general theory of syntax, abstracting away from details, which would provide the vehicle for this. Frankly, we do not know how to remedy this. What we will do instead, in Section 8 below, is look at some ways in which semantic and syntactic accounts of information can be merged.

\subsection{Proofs and information dynamics}

By far the most sophisticated paradigm bringing out information flow in inference and computation, as we have noted already, is logical Proof Theory. Proof systems are a form of 'discourse syntax', generating ordered tree structures of sentences with their inferential links indicated, and hence they are a natural candidate for representing information structure at a syntactic level.

Natural deduction and type theory In particular, the widely used method of natural deduction is an elegant calculus putting together, linking, and repackaging pieces of evidence with their dependencies. A paradigmatic illustration are its proof rules for the basic logical operation of conditional assertion:
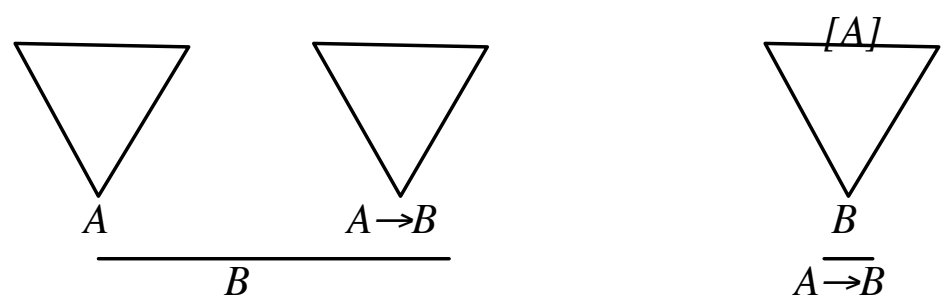

The view of mathematical proof calculi as general systems of combining evidence for claims has been put forward forcefully since the 1970s by authors such as Dummett, Martin-Löf, and Prawitz (cf. Sundholm 1986). Moreover, there are

\footnotetext{
${ }^{44}$ Similar distinctions have even been used for defining the classical philosophical 'analytic/synthetic' distinction as a matter of first-order logic.

${ }^{45}$ Hintikka's specific numerical proposals have been criticized ever since they appeared.
} 
natural notions of proof equivalence backing this up, for instance, through the valid identities of the lambda calculus and related abstract category-theoretic notions of equivalence (cf. Lambek \& Scott 1994). The resulting normal forms provide an attractive abstract level of information structure beyond brute details of syntax, and in the setting of linear logic, Girard 1987, 1989 has emphasized how proof normalization steps may be seen as communication moves, and hence as a form of information flow. We will not elaborate natural deduction, type theory, or category-theoretic proof paradigms in this chapter, but we refer to the chapter by Abramsky in this Handbook for an approach very much in the same spirit.

Proof construction as evidence dynamics Inference steps construct proofs, and hence they transform information at a syntactic level in a process of elucidation. This dynamics seems in line with the constructivist, or intuitionist slant of many proof theories (Dummett 1977, Troelstra \& van Dalen 1988), where one thinks of proof as performed by human agents creating objects and moving through successive information stages in the course of a mathematical enquiry. And even more dynamically, calculi of constructive proof can also be interpreted as models for multi-agent interaction. For instance, in the earlier-mentioned dialogue games of Lorenzen 1955, proof rules are game moves, and proofs are winning strategies for players. Viewed that way, proofs suddenly seem close to the dynamics we have placed at centre stage in this chapter. In this spirit, current 'logic of proofs' (starting from Artemov 1994) is developing a general take on evidence combination, and one can view the 'labeled deductive systems' of Gabbay 1996 as a program with similar ambitions. We will briefly return to both in Section 8 .

Even so, claims about the philosophical relevance of proof theory have a problem of 'transfer'. Mathematical proof is a very special way of establishing knowledge - and proofs, however elegant, hardly seem a paradigm for all the different sorts of evidence that humans manipulate, or the different ways in which they do so. Clearly, we are not just playing what Hintikka 1973 called 'indoor games' of proof or argumentation. Impeccable evidence also comes from our senses, our memory, from being told, and so on. And these other sources were described rather well in our earlier semantic accounts of information, without any obvious recasting as proof or computation. Therefore, a more promising line seems to be how to merge proof-theoretic and model-theoretic views of information into one story about rational agents capable of obtaining and integrating both.

Digression: logic programs As we said before, there are other proof formats which can represent information flow in inference: $\mathrm{cf}$. the extensive discussion in 
Allo 2007. In particular, Jago 2006 shows how logic programming, too, provides attractive models for inferential information flow. Here information states are sets of propositional literals $(\neg) p$, and information generating events are applications of Horn-clause rules $p_{1} \& \ldots, \& p_{n} \square q$, which let these states grow step by step. This dynamics can be made explicit by taking proof rules themselves as action expressions, and describing inference steps with modal operators $\langle$ rule $\rangle \square{ }^{46}$

In Section 8 we will discuss how proof-theoretic or other syntactic formats for information link up with our earlier semantic views. That there can be a significant connection seems clear from Gödel's completeness theorem which started off modern logic by showing how, in major systems like first-order logic, a formula $\square$ is true in all semantic models if and only if $\square$ has a syntactic proof.

\subsection{Agent orientation once more!}

Proof theory is often considered the most upper-class part of logic, dealing with crystalline mathematical structures where all traces of real human reasoning have been removed. But a return to the real world occurs as soon as we ask what good the information does which is created and transformed by inference. Syntax is clearly a representation of information as used by some agent, including possible idiosyncracies of formulation, and useful only given particular powers for performing elucidation on her data. What is informative for me in this manner need not be informative for you. Thus, deduction seems informative only for people engaged in certain tasks, and we are back with the central role of agency which also emerged in the earlier sections on agents construed semantically. This agent-orientation seems the right setting for understanding how an inference can inform us, and sometimes even surprise us, contradicting previous beliefs.

\section{Section 8 Semantics, Syntax and Proof: Merging Perspectives}

Even though syntactic and semantic views of information seem far apart in spirit, the recent literature has seen a number of attempts toward combining them. While there is no consensus on how to do this, or what can be achieved, we group a few approaches in this section, to introduce the reader to at least some challenges.

\subsection{Two-level syntax-semantics formats}

One kind of proposal, in various guises, combines semantic and syntactic views. Suppose that information states of agents really have two different levels: (a) the

\footnotetext{
${ }^{46}$ Like Égre 2006, Jago also views Fregean Morning Star / Evening Star puzzles with substituting identicals in intensional contexts as sources of non-omniscience and true inferential information.
} 
current range of models or possible worlds, and (b) some current set of sentences true in these models which the agent has available right now. Like the worlds in epistemic logic, and the situations of situation theory, the syntax domain, too, may be structured - say by an ordering of formulas as to relative importance in our current set of beliefs, or our current 'theory' (Ryan 1991, Gärdenfors 1987). Then we can think of informational processes as operating on two levels:

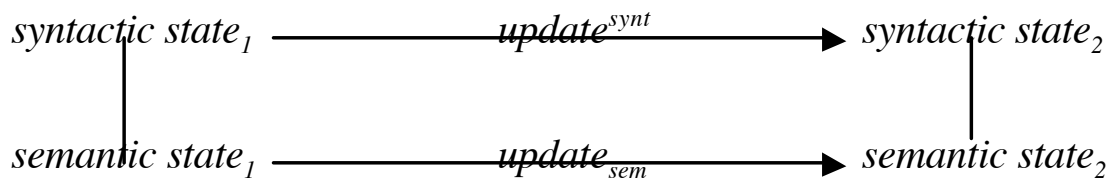

Range-changing updates modify the lower level, while inference modifies the upper syntax component of the current state, and hence the information 'immediately present' to the agent. This process can be described in the same sort of dynamic logic format used for epistemic logic in Section 3. Examples of this sort of approach include Dung 1995 on dynamic logic of argumentation, Wassermann 2001 on belief revision over syntactic knowledge bases, Gabbay, Kempson \& Meyer Viol 2001 on dynamic logic of grammars, and the earliermentioned Jago 2006. ${ }^{47}$ Two- and many-level views also occur at the interface of logic and cognitive science (Castelfranchi \& Lorini 2007) in realistic modeling of the many cognitive processes involved when humans process information.

\subsection{Merging calculi of proofs and evidence}

There are several ways of merging epistemic and proof-theoretic paradigms. Section 7 mentioned current attempts at broadening standard proof-theoretic accounts of internal elucidation with external observation of new information. We fist discuss one strand in the recent literature which stays close to proof theory.

Range and evidence: a case of $\square$ versus $\square$ For a start, as we have said earlier, proof-theoretic and semantic perspectives on information are not in conflict: they complement each other. But how? Let us first high-light a prima facie logical difference. As we saw in Section 2, epistemic logic of information as range revolves around a universal quantifier: $K_{i} \square$ says that

$\square$ is true in all situations agent $i$ considers as candidates for the current $s$.

\footnotetext{
${ }^{47}$ Also relevant are current syntax-flavoured 'neighbourhood models' for epistemic notions, such as the treatment of beliefs over time in Arlo-Costa \& Parikh 2005.
} 
But there is also an existential quantifier, occurring in the famous traditional account of knowledge that $\square$ as 'justified true belief. The first phrase "justified" in this definition says intuitively that

there exists a justification for the formula $\square$.

In this sense, knowledge consists in having evidence for a proposition: sometimes, on an exceptionally clear Cartesian day, even a mathematical proof. But as we saw, the two views co-exist in logic! Completeness theorems establish an equivalence between validity (an $\square$-type notion) and provability (an $\square$-type notion).

Combined calculi To have both views together then, van Benthem 1993 proposed merging epistemic logic with a 'calculus of evidence'. Candidates range from intuitionistic logic with binary type-theoretic assertions of the form

$x$ is a proof for $\square$.

to the much more general 'labeled deductive systems' of Gabbay 1996, which were designed as a general calculus of statements $x$ : $\square$, where the label $x$ can stand for a proof, an observation, or indeed any kind of evidence from any source, possibly even with further information attached about reliability, probability, and so on, of the support that $x$ offers to $\square$.

Even closer to the epistemic logics of Sections 2, 3, the 'logic of proofs' of Artemov 1994, 2005 is a modal language enriched with proof- or evidence-labeled assertions $[x] \square$. Then, e.g., the ubiquitous (though philosophically controversial; Egré 2004) epistemic Distribution Axiom $K_{i}(\square \square \square) \square \quad\left(K_{i} \square \square \quad K_{i} \square\right)$ becomes the more informative statement that

$$
[x](\square \square \square) \&[y] \square \square \quad[x \# y] \square,
$$

where \# is some natural sum operation on proofs, or pieces of evidence generally. Incidentally, van Benthem 2006A shows how this also works for contextualist views of information - as discussed in the chapter by Dretske in this Handbook. In that case the crucial law is

$$
\left[c_{1}\right] K_{i}(\square \square \square) \&\left[c_{2}\right] K_{i} \square \square \quad\left[c_{1} \# c_{2}\right] K_{i} \square,
$$

where \# is now an operation of 'context merge' reminiscent of situation theory. This is all fine, but it has to be admitted that one coherent philosophical interpretation of these combined systems still remains to be found. ${ }^{48}$

${ }^{48}$ For a merge of logic of proofs with dynamic epistemic logic, cf. Renne 2007.

Also relevant is Artemov 2007 on connections with general epistemology. 


\subsection{Merging proof and observation: two-level dynamic logics}

But integrating inferential information flow with observational update can also be done in formats that stay close to the dynamic logic of our Section 4. Here is a toy illustration from van Benthem 2007C, joining two simple components.

Consider the earlier logic programs for a propositional language with sets of literals that can be modified by inferential steps. Let observations be incoming hard information, as in the logic $P A L$ of public announcements. We need to hook up the syntactic information states with semantic ones. Here is one way of doing this. A semantic model $\boldsymbol{M}$ has valuations $V$ accepting or rejecting each proposition letter, i.e., complete worlds for our simple language. Next, $\boldsymbol{M}$ specifies a family of inference rules $\boldsymbol{R}$ whose corresponding conditionals are valid in the given set of valuations. Finally, it has partial sets of literals $X$ which do not yet decide every proposition letter. Each such set $X$ stands in an obvious 'compatibility relation' with all total valuations $V$ containing it. More precisely then, we can say that information states are pairs $(V, X)$ with $X$ a set of literals validated by $V$.

One can think of these as an agent's current semantic range about the empirical facts in the actual world plus its current inferential approximation to the worlds in that range. A matching logical language will access both components. Here a knowledge operator $K \square$ will operate on $V$ components only, referring to the whole semantic range as before. Next, one could access the $X$ component by an operator $I \square$ saying that the agent 'realizes that $\square^{k}$ if the literal $\square$ is actually present in $X$. In this setting, there are then several natural kinds of informative action:

Internal inference steps do not change the $V$ component of the current state, but take some rule and add literals to the current $X$ component (if the premises are there). For external observation we take $P A L$-style public announcements !P, removing all states $(V, X)$ where $V$ fails to satisfy $P$. But the interesting point of the combination is that there may be other 'intermediate' actions with something of both. In particular, think of the act of seeing in some conscious sense. We can model this with a third kind of update action on our hybrid information states. Explicit observations $+q$ of literals $q$ operate like announcements ! $q$, but then also place $q$ directly into all $X$ components of pairs $(V, X)$ in the remaining model. Putting all this into a logical system with both static and dynamic modalities, we 
can make combined assertions which describe the fine-structure of all these informational processes, both inferential and observational, within one setting. ${ }^{49}$

\subsection{Abstract viewpoints: toward a deeper unification?}

The preceding two approaches shows that evidence based $\square$-type and range-based $\square$-type accounts of information can live together. But this compatibility does not answer the more ambitious question if there is some deeper identity between evidence combination in an epistemic sense and that of syntax with proof theory. Stated as an observation about information and computation in general, proof theory and dynamic logics often address very similar issues and provide attractive solutions, whether in process theory or information dynamics, but the precise analogy between these two broad styles of thinking remains a bit of a mystery.

Abstract information theories: relevant and categorial logics Indeed, there are several abstract perspectives merging the two perspectives. One is that of Scott Information Systems, discussed by Michael Dunn in this Handbook. Another line are logics in the tradition of categorial and relevant logic, which have often been given an informational interpretation. One example is the 'Gaggle Theory' of Dunn 1991, inspired by the algebraic semantics for relevant logic, which provides an abstract framework that can be specialized to combinatory logic, lambda calculus and proof theory, but on the other hand to relational algebra and dynamic logic, i.e., the modal approach to informational events. Van Benthem 1991 arrives at a similar duality starting from categorial grammars for natural language, which sit at the interface of parsing-as-deduction and dynamic semantics. Indeed, he points out how the basic laws of the categorial 'Lambek Calculus' for product and directed implications have both dynamic and informational interpretations:

$$
\begin{array}{lll}
A \bullet B \square C & \text { iff } & B \square A \square C \\
A \bullet B \square C & \text { iff } & A \square C \square B
\end{array}
$$

Here, the product can be read dynamically as composition of binary relations modeling transitions of some process, and theimplications as the corresponding right- and left-inverses. But these laws can be read equally well as describing a universe of information pieces which can be merged by the product operation.

\footnotetext{
${ }^{49}$ Jago 2007 presents an alternative take on this, however, enriching the set of possible worlds with worlds carrying 'non-standard valuations' which are eliminated successively from the set of candidates through inferential steps. This seems a full-fledged alternative to the above pair-approach, based on ideas from relevant and paraconsistent logic (cf. Priest 1997).
} 
E.g., we can read $A \square B$ as the directed implication denoting $\{x \mid \square y \square A: y \bullet x \square B\}$, with $B \square A$ read in the obvious corresponding left-adjoining manner. On both interpretations, the principles of the Lambek Calculus hold (cf. van Benthem 1991 for further theory). Beyond that, however, the usual structural rules of classical inference turn out to fail ${ }^{50}$, and thus, there is a strong connection between substructural logics and what might be called abstract information theory (Mares 1996, Restall 2000, Mares 2003). This dynamic/informational interpretation also makes sense for Gabbay's earlier-mentioned paradigm of 'labeled deductive systems'. ${ }^{51}$ Sequoiah-Grayson 2007 is a spirited modern defense of the Lambek calculus as a minimal core system of information structure and information flow. While this is appealing, it has to be said that the above axioms merely encode the minimal properties of mathematical adjunctions, and these are so ubiquitous that they can hardly be seen as a substantial theory of information. ${ }^{52}$

Remark: Situation Theory after all Interestingly, authors in the relevant logic tradition also tend to see their systems as a direct continuation of the abstract analysis of information flow in situation theory - a link which we do not pursue here. The doctoral thesis Allo 2007 is an illuminating discussion of logical models underpinning the 'philosophy of information' set forth in the chapter by Floridi in this Handbook, joining up epistemic logic with sub-structural logics (Restall 2000), and Batens' 'adaptive logic' program (cf. Primero 2007).

But we are far from having exhausted all existing abstract information theories.

\footnotetext{
${ }^{50}$ In particular, the rules of Contraction and Permutation would express highly questionable assumptions about procedural or informational resources, which have no appeal in general.

${ }^{51}$ Van Benthem 1998 points out how semantic and proof-theoretic strands come together in labeled deductive systems. He unifies both in terms of a ternary calculus of abstract combination, with the following two rules: (a) $x: A, y: A \square B, R z, x y \mid-z: B$, where $R z, x y$ is some ternary condition relating $z, x, y$. Here the atom $R z, x y$ can be read as ' $z$ is the sum of the information pieces $x, y^{\prime}$, ' $z$ results from composing transitions $x, y^{\prime}, ' z$ results from applying function $x$ to argument $y^{\prime}$, etc. Principle (a) is then the abstract analogue of the earlier Modus Ponens in natural logic. Here is the principle for the dual rule of Conditionalization: (b) $\square: X, x: A, R z, x y \mid-z: B$ implies $\square: X \mid-$ $y: A \square B$. So far, this is still an abstract format without a corresponding intuitive unification.

${ }^{52}$ Just to mention some quite different sources, the same laws arise in matrix algebra, theories of vector spaces in mathematical morphology, and 'spatial implications' in logics of security.
} 
Other abstract models for unification are found in the work on logical omniscience in Parikh 1987, 1995, and most recently Parikh 2007. These papers propose an abstraction level that tries again to explain how deduction can be informative. ${ }^{53}$ Other relevant work is found in the Czech Tradition, of which we mention Tichy 2004, Materna 2004, and Duzi, Jespersen \& Müller 2005. But we conclude with a few strands from the grand tradition of foundational research.

\subsection{Coda: intuitionistic logic and modal information models}

Let us step back for a moment from latter-day abstractions. Maybe the oldest and still quite attractive joint proof-theoretic and semantic view of information occurs in intuitionistic logic (Dummett 1977, Troelstra \& van Dalen 1988; cf. Section 7). Unlike epistemic logic, it is a calculus of 'implicit knowledge' (van Benthem 1993), where the very meanings of the standard logical constants are tied up with being 'known' or 'provable'. Intuitionistic logic found its original proof-theoretic explanation in natural deduction-style through the so-called Brouwer-HeytingKolmogorov interpretation. But it also has modal-style semantic models due to Tarski, Beth, and Kripke, where 'worlds' are information stages in some ongoing enquiry. This long-standing harmony suggests that in logical systems like this, inferential and semantic information are very close. This strong connection comes out even very forcefully in the 'full completeness' theorems of Abramsky \& Jagadeesan 1994, which do not just link provability to validity, but specific proofs to concrete objects in (category-theoretic) semantics.

From intuitionistic to modal logics of information Intuitionistic logic has a very specific view on valid inference, far beyond what one would want to endorse in a general information theory. Thus, attempts have been made at abstracting a more general view of information, retaining its attractive features. Van Benthem 1996B proposes a 'modal theory of information' over models of abstract information stages ordered by inclusion (cf. also the 'data semantics' of Veltman 1984, Landman 1986), and there are related recent approaches to abstract information modeling in Sequoiah-Grayson 2006. These views seem related to the earliermentioned categorial and relevant approaches, but much remains to be clarified.

Digression: information that and information how Merging semantic and proof-theoretic perspectives is of interest in other ways, too. Proof theory has a clear algorithmic aspect, which we have ignored here (but cf. the chapters by

\footnotetext{
${ }^{53}$ Parikh 2007 also has an extremely interesting account of knowledge as that which is revealed in observable Nash equilibria of a new kind of evaluation games for testing assertions.
} 
Abramsky, Adriaans and Grunwald \&Vitanyi in this Handbook on various algorithmic aspects of information). Indeed, ever since Euclid's "Elements", constructive proofs are both ways of seeing that a conclusion is true and methods for constructing the relevant objects. In type-theoretic calculi, proofs are definable function terms in some mathematical universe through the Curry-Howard isomorphism. This duality ties in with the famous distinction between knowledge that and knowledge how (Gochet 2006) in philosophy and computer science. The latter is about procedures and general cognitive skills. This also makes sense for information. As pointed out in Israel \& Perry 1991, this notion is not just about what things are like in some situation, but also how to get thing things done.

\subsection{Conclusion}

Despite the abundance of promising ideas recorded in Sections 7 and 8, there is no consensus on the precise integration of inferential and semantic views of information structure and information dynamics. Indeed, the chapter by Samson Abramsky in this Handbook highlights the contrast even more, distinguishing logic as describing informational dynamics (the main thrust of our semantic approaches) from logic as embodying dynamics, the more proof-theoretical view that logical denotations are themselves informational processes. In such a light, our earlier Tandem View of representation and process would reflect two fundamental faces of logic itself. However this may be, given the history of logic, semantic and inferential views of information may be irreducibly complementary stances, and it may be precisely their interplay which is needed to grasp the notion of information in its entirety.

\section{Conclusion: Logic as Information Theory}

This chapter has painted logic as a theory of information, with an actual or potential impact on many disciplines. This is not at all the standard outside view or self-image of the field, but it does seem a legitimate perspective. We have woven a story which puts many logical notions and results into one new line, but one rather different from a standard textbook presentation. Here are our major conclusions, or rather: our claims, in doing so.

First, to get the real picture of logical information, one needs to address the statics and dynamics in parallel, with intertwined accounts of information structure and the dynamic processes which manipulate the latter. Next, there are many such dynamic processes, which need not all reduce to one primitive: be it inference or update, and hence logical views of information come in complementary kinds. 
More specifically, we have re-interpreted epistemic logic as an information theory of range, knowledge, and observation-based update. Like classical information theories, this is not just a model of information, but also a calculus for computing information flow, witness the concrete procedures for model update, and the axiomatic dynamic-epistemic laws governing this. We also emphasized the essential role of agents, and the ways in which agents take information: alone, but much more importantly, in interaction with others. Information is studied best in a setting of many agents, communication, and perhaps even interactive games.

Next, we have high-lighted another major perspective on information, viz. its 'aboutness', and the matching situation-theoretic account of correlations and dependence between different parts of distributed systems. This provides a much richer view of how agents can actually access information, and why it is there for them to pick up and communicate in the first place. We have shown that, despite some common misconceptions, this situation-theoretic perspective is not in conflict with the epistemic one, but rather forms its natural complement. - and we have provided a number of merged systems doing justice to all aspects so far.

Finally, logic offers another major view of information as code through its proof systems and other syntactic calculi which elucidate implicitly available semantic information by computing on suitable data structures. Our view is that this is another informational stance in its own right, close to the worlds of algorithmics and computation. We have surveyed some ways in which the syntactic stance and its natural processes can live in harmony with the semantic ones.

Re-interpreting logical systems in this way raises further questions. What does the usual logical agenda mean in this light? Does it provide a calculus for information comparable to that of the powerful Shannon-style or Kolmogorovstyle information theories in this Handbook? Most standard results in logic are concerned with (a) expressive power and definability, (b) axiomatic completeness for proof systems, or (c) computational complexity of tasks like model checking or proof search. We are not claiming that all of these are equally important as general concerns about 'information', but we do think that our re-interpretation might shed some fresh light on what we are doing in our standard grooves.

Finally, there is the issue of Grand Unification. Can all stances toward information be unified in one framework? We do not think so. Some abstract unification of all logical information theories may always exist, ascending far enough into mathematical Heaven. But what is the distance to Earth, i.e., our cognitive practices? Indeed, we doubt if a Grand Unification is necessary. Having 
several complementary stances in a field is fruitful in itself. Just compare the interplay of semantic and algorithmic views of information running throughout this Handbook. What is good enough, instead of unification, both in practice and in theory, is a rigorous way of analyzing differences and possible merges between stances. And that the latter is feasible should be clear from our text.

\section{Further Topics and Broader Outreach}

Our presentation has focused on a few major senses of information in logic, and some systems showing how they work. This is by no means an exhaustive survey of all topics and issues, and several alternative chapters could have been written mapping out the territory differently. Just think of analogies and differences with Probability Theory, logic's neighbour, but also its closest rival in the analysis of information. Of the omissions that weigh most on our conscience, besides logic and probability (cf. Loewe, Pacuit \& Romeijn 2007 for some recent interfaces), we mention a few, with some highly unsystematic references:

- Information content and the methodology of science (cf. Kuipers 2000)

- Visual and other information carriers beyond language

(Allwein \& Barwise 1996, Kerdiles 2001, Aiello et al. 2007)

- $\quad$ Information and context (Buvac \& McCarthy 2004)

- Information, interaction, and games (Sevenster 2006)

We console ourselves with the thought that many of these themes are found in other guises in other chapters of this Handbook, in particular, the ones on language, philosophy, economic game theory, computation, and cognition.

\section{Acknowledgments}

This chapter was written by two authors. Maricarmen Martinez was responsible for the parts on situation theory, with some valuable textual contributions by John Perry - and by Johan van Benthem for the epistemic logic and the other parts. We thank our commentators David Israel and John Perry for their critical comments throughout. In addition, we thank the following people for their stimulating responses to various versions of this chapter: Pieter Adriaans, Patrick Allo, Marie Duzi, Branden Fitelson, Paolo Mancosu, Edwin Mares, Ron van der Meyden, Larry Moss, Rohit Parikh, José Saguillo, and Sebastian Sequoiah-Grayson. 


\section{References}

S. Abramsky, 2006, 'Socially Responsive, Environmentally Friendly Logic', in T. Aho \& A-V Pietarinen, eds., Truth and Games: Essays in Honour of Gabriel Sandu, Acta Philosophica Fennica, 17 - 45.

S. Abramsky and R. Jagadeesan, 1994, 'Games and Full Completeness for Multiplicative Linear Logic', Journal of Symbolic Logic 59, 543 - 57.

P. Aczel, 1988, Non-well-founded Sets, CSLI Publications, Stanford.

M. Aiello, I. Pratt-Hartmann \& J. van Benthem, eds., 2007, Handbook of Spatial Logics, Springer, Dordrecht.

V. Akman \& E. Tin, 1997, 'Situated Non-monotonic Temporal Reasoning with BABY-SIT', AI Communications, 10; 93-109.

P. Allo, 2007, On Logics ands Being Informative, Ph.D. Thesis, Faculty of Arts, Vrije Universiteit Brussel.

G. Allwein \& J. Barwise (eds.), 1996, Logical Reasoning with Diagrams, Oxford University Press, New York.

H. Arlo-Costa \& E. Pacuit, 2005, 'First-Order Classical Modal Logic: Applications in Logics of Knowledge and Probability'. In R. van der Meyden, ed., Theoretical Aspects of Rationality and Knowledge: Proceeding of TARK X, 262 - 278.

H. Arlo-Costa \& R. Parikh, 2005, 'Some Non-normal Logics of Knowledge without Logical Omniscience', Department of Philosophy, Carnegie-Mellon University, Pittsburgh.

S. Artemov, 1994, 'Logic of Proofs', Annals of Pure and Applied Logic $67,29-59$.

S. Artemov, 2005, 'Evidence-Based Common Knowledge', CUNY Graduate Center, New York.

S. Artemov, 2007, 'Justification Logic', CUNY Graduate Center, New York.

A. Baltag, 1999, 'STS: A Structural Theory of Sets', Logic Journal of the ILPG 7 (4), 481-515.

A. Baltag, J. van Benthem \& S. Smets, 2007, ‘A Dynamic-Logical Approach to Interactive Epistemology', Working Paper, Institute for Logic, Language and Computation, Amsterdam.

A. Baltag, L. Moss \& S. Solecki, 1998, 'The Logic of Public Announcements, Common Knowledge and Private Suspicions', Proceedings TARK 1998, 43 - 56, Morgan Kaufmann Publishers, Los Altos.

A. Baltag \& S. Smets, 2006, 'Dynamic Belief Revision over Multi-Agent Plausibility Models', Proceedings LOFT 2006, Department 
of Computing, University of Liverpool.

A. Baltag \& S. Smets, 2007, 'Logics for Quantum Information Flow', Workshop Philosophy of Information, Oxford University.

To appear in Knowledge, Rationality and Action.

H. Barendregt, 1984, The Lambda Calculus, its Syntax and Semantics, North-Holland, Amsterdam.

Y. Bar-Hillel \& R. Carnap, 1953, 'Semantic Information', The British Journal for the Philosophy of Science 4:14, 147 - 157.

J. Barwise, 1997, 'Information and Impossibilities', Notre Dame Journal of Formal Logic 38, 488 - 515.

J. Barwise \& J. van Benthem, 1999, 'Interpolation, Preservation, and Pebble Games', Journal of Symbolic Logic 64, 881-903.

J. Barwise \& J. Etchemendy, 1987, The Liar: An Essay on Truth and Circularity, Oxford University Press, New York.

J. Barwise \& L. Moss, 1996, Vicious Circles: On the Mathematics of Non-Wellfounded Phenomena, CSLI Publications, Stanford.

J. Barwise \& J. Seligman, 1997, Information Flow: The Logic of

Distributed Systems, Cambridge University Press, Cambridge.

J. Barwise \& J. Perry, 1983, Situations and Attitudes, The MIT Press, Cambridge (Mass.).

N. Belnap, 1982, 'Display Logic', Journal of Philosophical Logic 11, 375 - 417.

J. van Benthem, 1991, Language in Action: Categories, Lambdas, and Dynamic

Logic, North-Holland Amsterdam \& MIT Press, Cambridge (Mass.).

J. van Benthem, 1993, 'Reflections on Epistemic Logic', Logique et Analyse

$34,5-14$.

J. van Benthem, 1996A, Exploring Logical Dynamics, CSLI Publications,

Stanford.

J. van Benthem, 1996B, 'Modal Logic as a Theory of Information', in

J. Copeland, ed., Logic and Reality. Essays on the Legacy of Arthur Prior, Clarendon Press, Oxford, 135 - 168.

J . van Benthem, 1998, 'Proofs. Labels, and Dynamics in Natural Language', in U. Reyle \& H-J Ohlbach, eds., Festschrift for Dov Gabbay, 31-41, Kluwer Academic Publishers, Dordrecht.

J. van Benthem, 2000, 'Information Transfer Across Chu Spaces',

Logic Journal of the IGPL 8, $719-731$.

J. van Benthem, 2001, 'Games in Dynamic Epistemic Logic', 
Bulletin of Economic Research 53:4, 219 - 248.

J. van Benthem, 2005A, 'Guards, Bounds, and Generalized Semantics', Journal of Logic, Language and Information 14, 263 - 279.

J. van Benthem, 2005B, 'Information as Correlation versus Information as Range', Research Report, Institute for Logic, Language and Computation, University of Amsterdam. To appear in L. Moss, ed., Logic and Cognition, Memorial Volume for Jon Barwise.

J. van Benthem, 2006A, 'Epistemic Logic and Epistemology: the state of their affairs', Philosophical Studies 128, 49- 76.

J. van Benthem, 2006B, 'One is a Lonely Number: on the logic of communication', in Z. Chatzidakis, P. Koepke \& W. Pohlers, eds., Logic Colloquium '02, ASL \& A.K. Peters, Wellesley MA, 96 - 129.

J. van Benthem, 2007A, 'Dynamic Logic of Belief Revision', Journal of Applied Non-Classical Logics 17, 129 - 155.

J. van Benthem, 2007B, Inference in Action, Research Report, Institute for Logic, Language and Computation, University of Amsterdam. To appear in Logic in Serbia, Serbian Academy of Sciences, Beograd.

J. van Benthem, 2007C, '"Tell It Like It Is": Information Flow in Logic', Philosophical Review, Beijing.

J. van Benthem \& G. Bezhanishvili, 2007, 'Modal Logics of Space', in M. Aiello, I. Pratt \& J. van Benthem, eds., Handbook of Spatial Logics, Springer, Dordrecht, $217-298$.

J. van Benthem \& P. Blackburn, 'Modal Logic, a Semantic Perspective', in J. van Benthem, P. Blackburn \& F. Wolter, eds., Handbook of Modal Logic, Elsevier, Amsterdam, $1-84$.

J. van Benthem, J. van Eijck \& B. Kooi, 2006, 'Logics of Communication and Change', Information and Computation 204(11), 1620 - 1662.

J. van Benthem, J. Gerbrandy \& E. Pacuit, 2007, 'Merging Frameworks for Interaction: DEL and ETL', ILLC Amsterdam \& Informatics Torino. Proceedings TARK 2007, University of Namur.

J. van Benthem, D. Israel \& J. Perry, 2007, 'Situation Theory and Dynamic Logic', Department of Philosophy, Stanford University.

J. van Benthem \& F. Liu, 2004, 'Diversity of Logical Agents in Games', Philosophia Scientiae 8:2, 163 - 178.

J. van Benthem \& F. Liu, 2007, 'Dynamic Logics of Preference Upgrade', Journal of Applied Non-Classical Logics 17, 157 - 182.

J. van Benthem \& E. Pacuit, 2006, 'The Tree of Knowledge in Action', 
Proceedings Advances in Modal Logic, ANU Melbourne.

J. van Benthem \& D. Sarenac, 2005, 'The Geometry of Logic', in J-Y Béziau,

A. Costa Leite \& A. Facchini, eds., Aspects of Universal Logic,

Centre de Recherches Sémiologiques, Université de Neuchatel, 1 - 31.

S. Blamey, 2002, 'Partial Logic', in D. Gabbay \& F. Guenthner, eds.,

Handbook of Philosophical Logic, Vol. 5 (2nd ed.), Kluwer,

Dordrecht, 261-353.

B. de Bruin, 2004, Explaining Games, Dissertation. ILLC, University of Amsterdam.

J. Burgess, 1981, 'Quick Completeness Proofs for some Logics of Conditionals',

Notre Dame Journal of Formal Logic 22, 76 - 84.

S. Buvac \& J. McCarthy, 2004, 'Formalizing Context (Expanded Notes)', Technical

Report CS-TN-94-13, Department of Computer Science, Stanford University.

R. Carnap, 1952, The Continuum of Inductive Methods, The University of

Chicago Press, Chicago.

B. Chellas, 1980, Modal Logic: an Introduction, Cambridge University

Press, Cambridge.

J. Corcoran, 1998, 'Information-Theoretic Logic', in C. Martínez, U. Rivas,

L. Villegas-Forero, eds., Truth in Perspective,

Ashgate Publishing, Aldershot, 113-135.

D. van Dalen, 2002, 'Intuitionistic Logic', in D. Gabbay \& F. Guenthner, eds.,

Handbook of Philosophical Logic, Vol. 5 (2nd ed.), Kluwer,

Dordrecht, 1-114.

Devlin, K., 1991, Logic and Information, Cambridge University Press.

H. van Ditmarsch, W. van der Hoek \& B. Kooi, 2007, Dynamic Epistemic Logic,

Springer. Dordrecht.

K. Doets, 1994, From Logic to Logic Programming, The MIT Press,

Cambridge (Mass.).

F. Dretske, 1981, Knowledge and the Flow of Information,

The MIT Press, Cambridge (Mass.).

M. Dummett, 1977, Elements of Intuitionism, Oxford University Press, Oxford.

P. Dung, 1995, ‘An Argumentation-Theoretic Foundation for Logic

Programming', Journal of Logic Programming 22, 151 - 177.

M. Dunn, 1991, 'Gaggle Theory: An abstraction of Galois connections and

Residuation, with applications to negation, implication, and various

logical operators', in J. van Eijck, ed., Logics in AI (Amsterdam,

1990), Springer, Berlin, $31-51$.

M. Duzi, B. Jespersen \& B. Müller, 2005, 'Epistemic Closure and Inferable 
Knowledge', in L. Behounek \& M. Bilkova, eds., The Logica

Yearbook 2004, Filosofia, Prague, 125 - 140.

P. Egré, 2004, Attitudes propositionnelles et paradoxes épistémiques

Thèse de Doctorat, Université Paris 1 Panthéon-Sorbonne, IHPST.

R. Fagin, J. Halpern, Y. Moses \& M. Vardi, 1995, Reasoning about Knowledge, The MIT Press, Cambridge (Mass.).

S. Feferman, 2000, 'Highlights in Proof Theory', in V. Hendricks et al., eds., Proof Theory, pages 11 - 31. Kluwer Academic Publishers, Dordrecht.

B. Fitelson, 2006, 'Old Evidence, Logical Omniscience \& Bayesianism', Lecture ILLC Workshop Probability and Logic, Department of Philosophy, University of California at Berkeley.

M. Forti \& F. Honsell, 1983, 'Set Theory with Free Construction Principles', Annali della Scuola Normale Superiore di Pisa, Classe di Scienze, Serie IV:10, 493 - 522.

D. Gabbay, 1996, Labeled Deductive Systems, Oxford University Press, Oxford.

D. Gabbay, R. Kempson, \& W. Meyer-Viol, 2001, Dynamic Syntax:

The Flow of Language Understanding, Blackwell, Oxford.

B. Ganter \& R. Wille, 1997, Formal Context Analysis: Mathematical Foundations, Springer Verlag, New York.

P. Gärdenfors, 1987, Knowledge in Flux, on the Dynamics of Epistemic States, The MIT Press, Cambridge (Mass.).

P. Gärdenfors \& H. Rott, 1995, 'Belief Revision', in D. M. Gabbay, C. J. Hogger \& J. A. Robinson, eds., Handbook of Logic in Artificial Intelligence and Logic Programming 4, Oxford University Press, Oxford 1995.

J. Gerbrandy, 1999, Bisimulations on Planet Kripke, Dissertation, Institute for Logic, Language and Computation, University of Amsterdam.

C. Ghidini \& F. Giunchiglia, 2001, 'Local Model Semantics, or Contextual Reasoning = Locality + Compatibility', Artificial Intelligence 127, $221-259$.

J. Gibson, 1986, The Ecological Approach to Visual Perception, Lawrence Erlbaum Associates, Hillsdale, NJ.

J-Y Girard, 1987, 'Linear Logic', Theoretical Computer Science 50, 1 - 102.

J-Y Girard, 1989, 'Towards a Geometry of Interaction', in J. W. Gray and A. Scedrov, eds., Categories in Computer Science and Logic, American Mathematical Society, 69 - 108.

P. Gochet, 2006, 'La Formalisation du Savoir-faire', Lecture at Pierre Duhem Colloquium IPHRST Paris, Philosophical Institute, 
Université de Liege.

V. Gupta, 1994, 'Chu Spaces: A Model of Concurrency', PhD Thesis, Stanford University, September 1994.

J. Halpern \& M. Vardi, 1989, 'The Complexity of Reasoning about Knowledge and Time', Journal of Computer and System Sciences 38, 195 - 237.

V. Hendricks, 2006, Mainstream and Formal Epistemology, Cambridge University Press, New York.

J. Hintikka, 1962, Knowledge and Belief, Cornel University Press, Ithaca.

J. Hintikka, 193, Logic, Language-Games and Information, Clarendon Press, Oxford.

W. van der Hoek \& J-J Meijer, 1995, Epistemic Logic for AI and Computer Science, Cambridge University Press, Cambridge.

W. van der Hoek \& M. Pauly, 2006, 'Modal Logic for Games and Information', in P. Blackburn, J. van Benthem \& F. Wolter, eds., Handbook of Modal Logic, Elsevier, Amsterdam, 1077 - 1148.

D. Israel \& J. Perry, 1990, 'What is Information? ', In: P. Hanson (ed.). Information, Language and Cognition. University of British Columbia Press, Vancouver.

D. Israel \& J. Perry, 1991, 'Information and Architecture'. In: J. Barwise, J. M. Gawron, G. Plotkin, and S. Tutiya (eds.). Situation Theory and Its Applications, vol. 2. CSLI Publications, Stanford.

B. Jacobs \& J. Rutten, 1997, 'A Tutorial on (Co)Algebras and (Co)Induction', EACTS Bulletin 62, 222-259.

M. Jago, 2006, Logics for Resource-Bounded Agents, Dissertation, Department of Philosophy, University of Nottingham.

M. Jago, 2007, 'Logical Information is Vague', Department of Philosophy, University of Nottingham, to appear in Knowledge, Rationality and Action.

K. Kelly, 1996, The Logic of Reliable Inquiry, Oxford University Press, New York.

G. Kerdiles, 2001, Saying It with Pictures: a Logical Landscape of Conceptual Graphs, Dissertation, Institute for Logic, Language and Computation, University of Amsterdam.

M. Knauff, 2007, 'How our Brains Reason Logically', Topoi: an International Review of Philosophy 26, 19 - 36.

R. Kowalski, 1979, Logic for Problem Solving, North-Holland, New York. A. Kratzer, 2006, 'Situations in Natural Language Semantics', the 
Stanford Encyclopedia of Philosophy, http://www.plato.stanford.edu

Th. Kuipers, 2000, From Instrumentalism to Constructive Realism,

Kluwer, Dordrecht.

J. Lambek \& Ph. Scott, 1994, Introduction to Higher-Order Categorical

Logic, Cambridge University Press, Cambridge.

F. Landman, 1986, Towards a Theory of Information, the Status of

Partial Objects in Semantics, Foris, Dordrecht,.

D. Lewis, 1969, Convention, A Philosophical Study, Harvard University

Press, Harvard.

D. Lewis, 1970, 'General Semantics', Synthese 22, 18 - 67.

F. Liu, 2006, 'Diversity of Agents', Research Report, Institute for Logic,

Language and Computation, Amsterdam. To appear in

Journal of Logic, Language and Information.

F. Liu \& J. Zhang, 2007, 'Some Thoughts on Mohist Logic', in J. van Benthem,

S. Ju \& F. Veltman, eds., A Meeting of the Minds, Proceedings LORI

Beijing 2007, College Publications, London, 79 - 96.

B. Loewe, E. Pacuit and J-W Romeijn, 2007, Foundations of the Formal

Sciences VI: Reasoning about Probabilities and Probabilistic Reasoning,

Institute for Logic, Language and Computation, University of Amsterdam.

P. Lorenzen, 1955, Einführung in die Operative Logik und Mathematik,

Springer, Berlin.

E. Lorini \& C. Castelfranchi, 2007, 'The Cognitive Structure of Surprise: looking for basic principle's, Topoi 2007; 133 - 149.

E. Mares, 1996, 'Relevant Logic and the Theory of Information', Synthese 109, $345-360$.

E. Mares, 2003, Relevant Logic, a Philosophical Interpretation, Cambridge University Press, Cambridge.

M. Martinez, 2004, Commonsense Reasoning via Product State Spaces, Ph.D. Thesis, Indiana University, Bloomington, USA.

P. Materna, 2004, Conceptual Systems, Logos Verlag, Berlin.

R. Moore, 1985, ‘ A Formal Theory of Knowledge and Action'. In J. Hobbs $\&$ R. Moore, eds., Formal Theories of the Commonsense World, Ablex Publishing Corp, pp. 319-358.

R. Moore, 1989, 'Propositional Attitudes and Russellian Propositions', in R. Bartsch, J. van Benthem and P. van Emde Boas, eds., Semantics and Contextual Expression, Foris, Dordrecht, 147 - 174.

L. Moss, R. Parikh \& Ch. Steinsvold, 2007, 'Topology and Epistemic Logic', 
in M. Aiello, I. Pratt \& J. van Benthem, eds., Handbook of Spatial Logics, Springer, Dordrecht, 299 - 341.

L. Moss \& J. Seligman, 1997, 'Situation Theory', in J. van Benthem \&

A. ter Meulen, eds., Handbook of Logic and Language,

North Holland, Amsterdam.

I. Németi, 1995, 'Decidable Versions of First-Order Logic and Cylindric-

Relativized Set Algebras', in L. Csirmaz, D. Gabbay \& M. de Rijke, eds., 1995, Logic Colloquium 92.Veszprem, Hungary, Studies in Logic,

Language and Information, CSLI Publications, Stanford, 177-241.

M. Osborne \& A. Rubinstein, 1994, A Course in Game Theory,

The MIT Press, Cambridge (Mass.).

Ch. Papadimitriou, 1994, Computational Complexity, Addison-Wesley, Reading (Mass.).

R. Parikh, 1987, 'Knowledge and the Problem of Logical Omniscience',

ISMIS-87, International Symposium on Methodology for

Intelligent Systems, North-Holland, 432 - 439.

R. Parikh, 1995, 'Logical Omniscience', in D. Leivant, ed., Logic

and Computational Complexity, Springer, Heidelberg, 22 - 29.

R. Parikh, 2007, 'Sentences, Propositions, and Group Knowledge',

Lecture at First Synthese Annual Conference Copenhagen,

CUNY Graduate Center, New York.

R. Parikh \& R. Ramanujam, 2003, 'A Knowledge Based Semantics of

Messages', Journal of Logic, Language and Information 12, 453 - 467.

D. Prawitz, 1965, Natural Deduction. A Proof-Theoretical Study,

Almqvist and Wiksell, Stockholm.

G. Priest, 1997, 'Impossible Worlds - Editor's Introduction', Notre Dame

Journal of Formal Logic 38, 481 - 487.

G. Primero, 2007, 'Belief Merging Based on Adaptive Interaction', in J. van

Benthem, S. Ju \& F. Veltman, eds., A Meeting of the Minds,

Proceedings LORI Beijing 2007, College Publications London, 315 - 320.

H. Putnam, 1981, Reason, Truth and History, Cambridge University Press.

B. Renne, 2007, 'Public Communication in Justification Logic',

CUNY Graduate Center, New York.

G. Restall, 2000, An Introduction to Substructural Logics, Routledge, London.

H. Rott, 2006, 'Shifting Priorities: Simple Representations for Twenty-Seven

Iterated Theory Change Operators', Philosophical Institute,

University of Regensburg. 
W. C. Rounds, 1997, 'Feature Logics', in J. van Benthem and A. ter Meulen, eds., Handbook of Logic and Language, Elsevier, Amsterdam, $475-533$.

Sh. Roush, 2006, Tracking Truth: Knowledge, Evidence and Science, Oxford University Press, Oxford.

M. Ryan, 1991, Defaults and Revision in Structured Theories, Dissertation, Department of Computing, Imperial College, London.

J. Saguillo, 1997, 'Logical Consequence Revisited', The Bulletin of Symbolic Logic 3:2, 216-241.

D. SanGiorgi, 2004, 'Bisimulation: From The Origins to Today', Proceedings LICS, 298 - 302, Extended new version, 2007, Department of Informatics, University of Bologna.

H. Schwichtenberg \& A. Troelstra, 1996, Basic Proof Theory, Cambridge University Press, Cambridge.

S. Sequoiah-Grayson, 2006, 'Information Flow and Impossible Situations', Logique et Analyse 196, 371 - 398.

S. Sequoiah-Grayson, 2007, 'Information Gain from Inference', Philosophical Institute, Oxford. To appear in Knowledge, Rationality and Action.

M. Sevenster, 2006, Branches of Imperfect Information, Dissertation, Institute for Logic, Language and Computation, University of Amsterdam.

F. Staal, 1988, Universals: Studies in Indian Logic and Linguistics, University of Chicago Press, Chicago and London.

G. Sundholm, 1986, 'Proof Theory and Meaning', in D. Gabbay \& F. Guenthner, eds., Handbook of Philosophical Logic III, Reidel, Dordrecht, 471 - 506.

N. Tennant, 1978, Natural Logic, Edinburgh University Press, Edinburgh.

P. Tichy, 2004, Collected Papers in Logic and Philosophy, V. Svoboda, B. Jespersen, \& C. Cheyne, eds., Filosofia, Prague \& University of Otago Press, Dunedin.

A. Troelstra \& D. van Dalen, 1988, Constructivism in Mathematics:

An Introduction, North-Holland Publishing, Amsterdam.

J. Väänänen, 2007, Dependence Logic: A New Approach to Independence Friendly Logic, Cambridge University Press, Cambridge.

F. Veltman, 1984, 'Data Semantics', in J. Groenendijk, Th. Janssen \& M. Stokhof, eds., Truth, Interpretation and Information, Foris, Dordrecht, $43-63$. 
F. Veltman, 1985, Logics for Conditionals, Dissertation, Philosophical Institute, University of Amsterdam.

Y. Venema, 2006, 'Algebras and Co-algebras', in J. van Benthem, P. Blackburn \& F. Wolter, eds., Handbook of Modal Logic, Elsevier, Amsterdam, $331-426$.

R. Wassermann, 2001, Resource Bounded Belief Revision, Dissertation, Institute for Logic, Language and Computation, University of Amsterdam.

T. Williamson, 2000, Knowledge and its Limits, Oxford University Press, Oxford. 